\title{
5-(5-Aryl-1,3,4-oxadiazole-2-carbonyl)furan-3-carboxylate and New Cyclic $C$-Glycoside Analogues from Carbohydrate Precursors with MAO-B, Antimicrobial and Antifungal Activities
}

\author{
Mohamed Mohamed El-Sadek ${ }^{1, *}$, Seham Yassen Hassan ${ }^{1}$, Nagwa Said Abd El-Dayem ${ }^{1}$ \\ and Galila Ahmed Yacout ${ }^{2}$
}

1 Chemistry Department, Faculty of Science, Alexandria University, Alexandria 21231, Egypt;

E-Mails: sehamyassen@yahoo.com (S.Y.H.); nagwa_abdeldayem@yahoo.com (N.S.A.E.-D.)

2 Biochemistry Department, Faculty of Science, Alexandria University, Alexandria 21231, Egypt;

E-Mail: galila_69@yahoo.com

* Author to whom correspondence should be addressed; E-Mail: elsadek_mm@yahoo.com;

Tel.: +20-01-006-544-617; Fax: +20-3-593-2488.

Received: 1 April 2012; in revised form: 27 April 2012 / Accepted: 9 May 2012 /

Published: 7 June 2012

\begin{abstract}
Cyclization of acyclic $C$-glycoside derivatives $\mathbf{1 a , b}$ to $\mathbf{2 a , b}$ as the major isomers, and $\mathbf{4} \mathbf{a}, \mathbf{b}$ as the minor isomers were carried out. The isopropylidene derivatives 3a,b were prepared, as well as the hydrazide derivative $\mathbf{6}$, which was condensed with a variety of aldehydes to give hydrazones $7 \mathbf{a}-\mathbf{e}$ which were also prepared from the compounds 12a-e. Acetylation of 7a,d gave the corresponding acetyl derivatives $\mathbf{8 a , d}$, respectively. In addition, the dicarbonyl compound $\mathbf{9}$ was prepared in the hydrate form, which reacted with a number of aroylhydrazines to give the corresponding bisaroylhydrazones 10a-d, which were cyclized into 1,3,4-oxadiazoles 11a-d. Furthermore, two of the prepared compounds were examined to show the ability to activate MAO-B. In addition a number of prepared compounds showed antibacterial and antiviral activities.
\end{abstract}

Keywords: carbohydrazone; isopropylidene; triazole; oxadiazole; monoamine oxidase-B 


\section{Introduction}

$C$-glycosides have received a great deal of attention from the synthesis and medicinal chemistry community, due to their increased stability to hydrolysis as well as their presence in a number of interesting natural products [1]. Furthermore, heterocyclic compounds containing the five-membered oxadiazole nucleus possess a diversity of useful biological effects. Substituted 1,3,4-oxadiazoles are of considerable pharmaceutical interest, for instance, 2-amino-1,3,4-oxadiazoles act as muscle relaxants [2] and show antimitotic activity. Anti-inflammatory [3,4], antimicrobial [4], anti-hepatitis B [5] and anti-diarrheal activity [6] of some new 1,3,4-oxadiazole derivatives was also reported. Recently several 1,3,4-oxadiazole derivatives were identified as potentially active antimycobacterial [7,8], antitubercular [9], anticonvulsant [10] and anticancer [11] agents, and also reported as enzyme tyrosinase inhibitors [12]. In light of these interesting biological activities, it became interested in synthesizing some new $C$-glycosides of substituted 1,3,4-oxadiazole derivatives and evaluating their antimicrobial potential.

\section{Results and Discussion}

\subsection{Chemistry}

It has been shown [13] that the acid-catalysed, intramolecular dehydration of $\mathbf{1 b}$ using conc. $\mathrm{HCl}\left(0^{\circ} \mathrm{C}\right)$ yields a mixture of a major D-ribo anhydro isomer $\mathbf{2 b}$ (with inversion of configuration), and a minor D-arabino-anhydro isomer $\mathbf{4 b}$ (with retention of configuration). On the other hand, the dehydration of 1a with aqueous acetic acid (10\%) under reflux [14], afforded a mixture of anhydro derivatives 2a (major isomer) and $\mathbf{4 a}$ (the minor one) and in addition, a mixture of $\mathbf{2 b}$ and $\mathbf{4 b}$ were obtained from $\mathbf{1 b}$ using aqueous acetic acid (10\%). The minor isomer $\mathbf{4 a}$ could be isolated as a solid.

The anomeric configurations [15] of these anhydro derivatives were ascertained from the ${ }^{1} \mathrm{H}-\mathrm{NMR}$ spectra of their isopropylidene derivatives $\mathbf{3 a , b}$ which were prepared by an improved procedure [16] through treatment of the crude anhydro derivatives with acetone in the presence of catalytic amount of $p$-toluenesulphonic acid (see Experimental and Scheme 1).

Scheme 1. Synthesis of isopropylidene derivatives 3a,b.

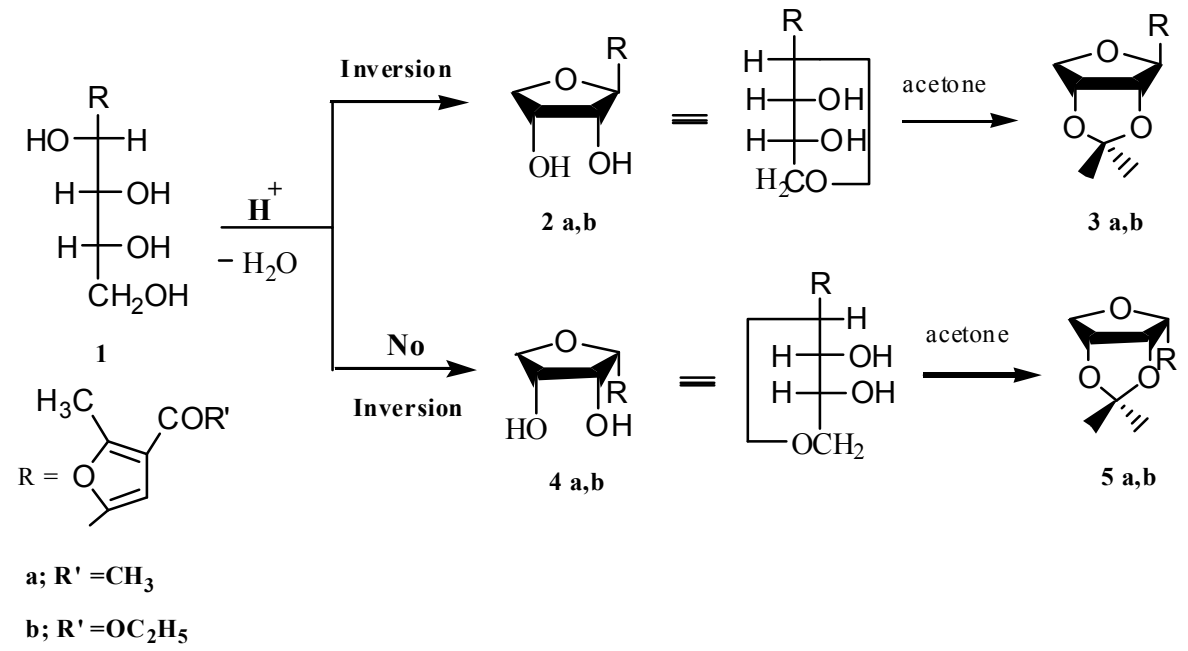


${ }^{1} \mathrm{H}-\mathrm{NMR}$ spectra $\left(\mathrm{CDCl}_{3}\right)$ of compounds 3a,b displayed the two methyl protons signals of the 2,2-dimethyldioxolane ring at $\delta(1.544-1.352)$ having $\Delta \delta 0.187$. The signals of the sugar protons of these isopropylidene derivatives were assigned from the $2 \mathrm{D}{ }^{1} \mathrm{H}-\mathrm{NMR}$ spectrum of compound $\mathbf{3 b}$ (Figure 1), and the characteristic chemical shifts as compared with those reported for anhydro analogues [16], whereby the $\mathrm{C}-1^{\prime}$ proton appears as a singlet at $\delta 5.00\left(J_{1^{\prime}, 2^{\prime}}=0.00 \mathrm{~Hz}\right)$. Confirmation of the anomeric configuration of the isopropylidene derivatives $\mathbf{3 a , b}$ can be obtained from the zero coupling constant value $\left(J_{1^{\prime}, 2^{\prime}}=0.00 \mathrm{~Hz}\right)$, which is an unequivocal [16,17] proof for the trans arrangement of the H-1' and H-2' ( $\beta$-D-configuration) as well as from the $\Delta \delta$ value $(0.187)[15,18,19]$. Boiling of the crude $\mathbf{2 b}, \mathbf{4 b}$ mixture with hydrazine hydrate resulted in the formation of $\mathbf{6}$, which upon condensation with a number of aldehydes afforded the corresponding anhydrohydrazone derivatives 7a-e which were also obtained as only one isomer (inversion of configuration at C-1') by boiling the compounds 12a-e [20,21] with aqueous acetic acid under reflux. The assignment of the signals for the sugar protons in the ${ }^{1} \mathrm{H}-\mathrm{NMR}$ spectra of compounds $7 \mathbf{a}-\mathbf{e}$ were based on the $2 \mathrm{D}{ }^{1} \mathrm{H}-\mathrm{NMR}$ spectrum of compound 7e. In addition, acetylation of the anhydrohydrazones 7a,d afforded the corresponding $O$-acetyl derivatives 8a,d, respectively (see Experimental and Scheme 2).

Figure 1. 2D ${ }^{1} \mathrm{H}-\mathrm{NMR}$ spectrum of compound $\mathbf{3 b}$.

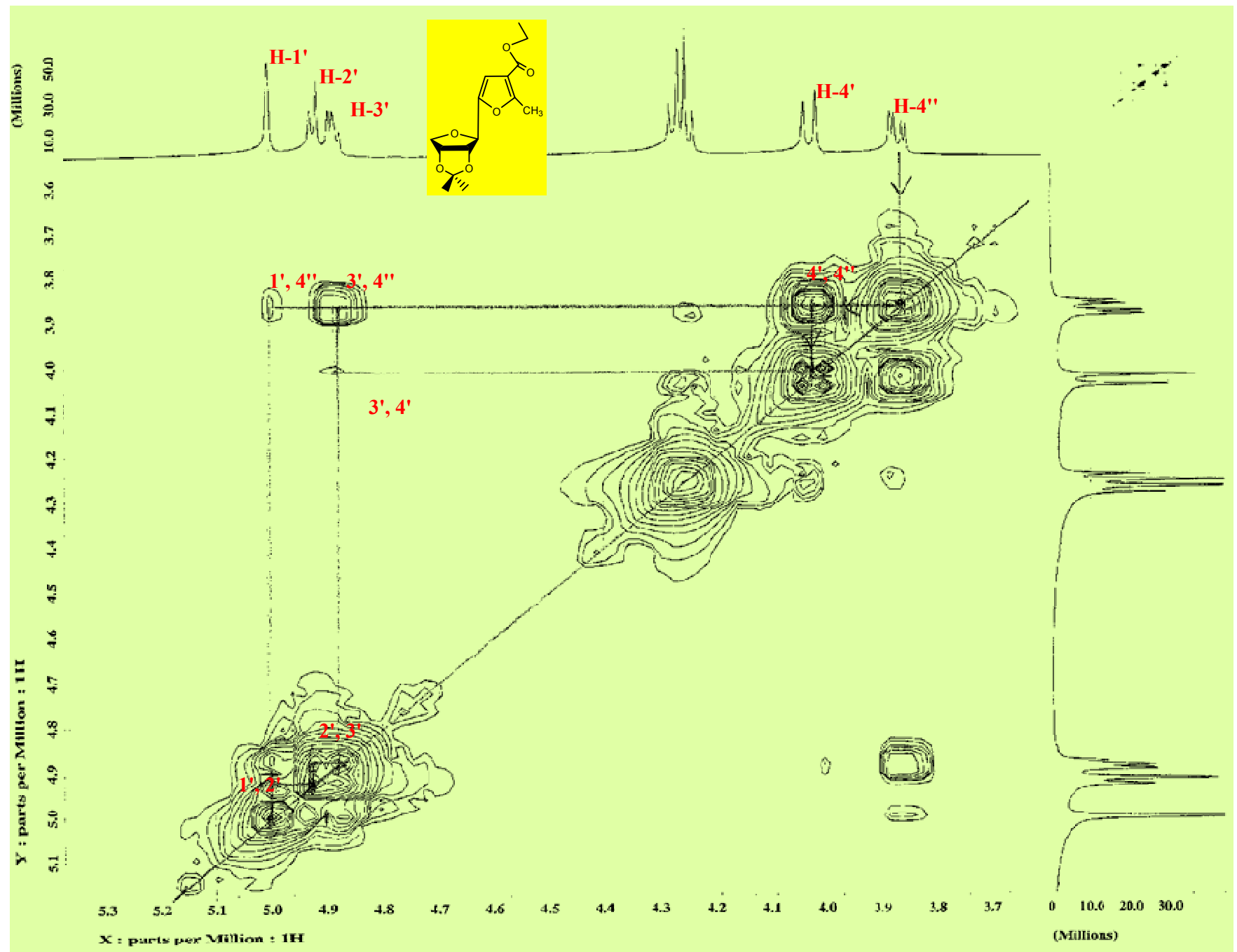


Scheme 2. Synthesis of carbohydrazone derivatives.

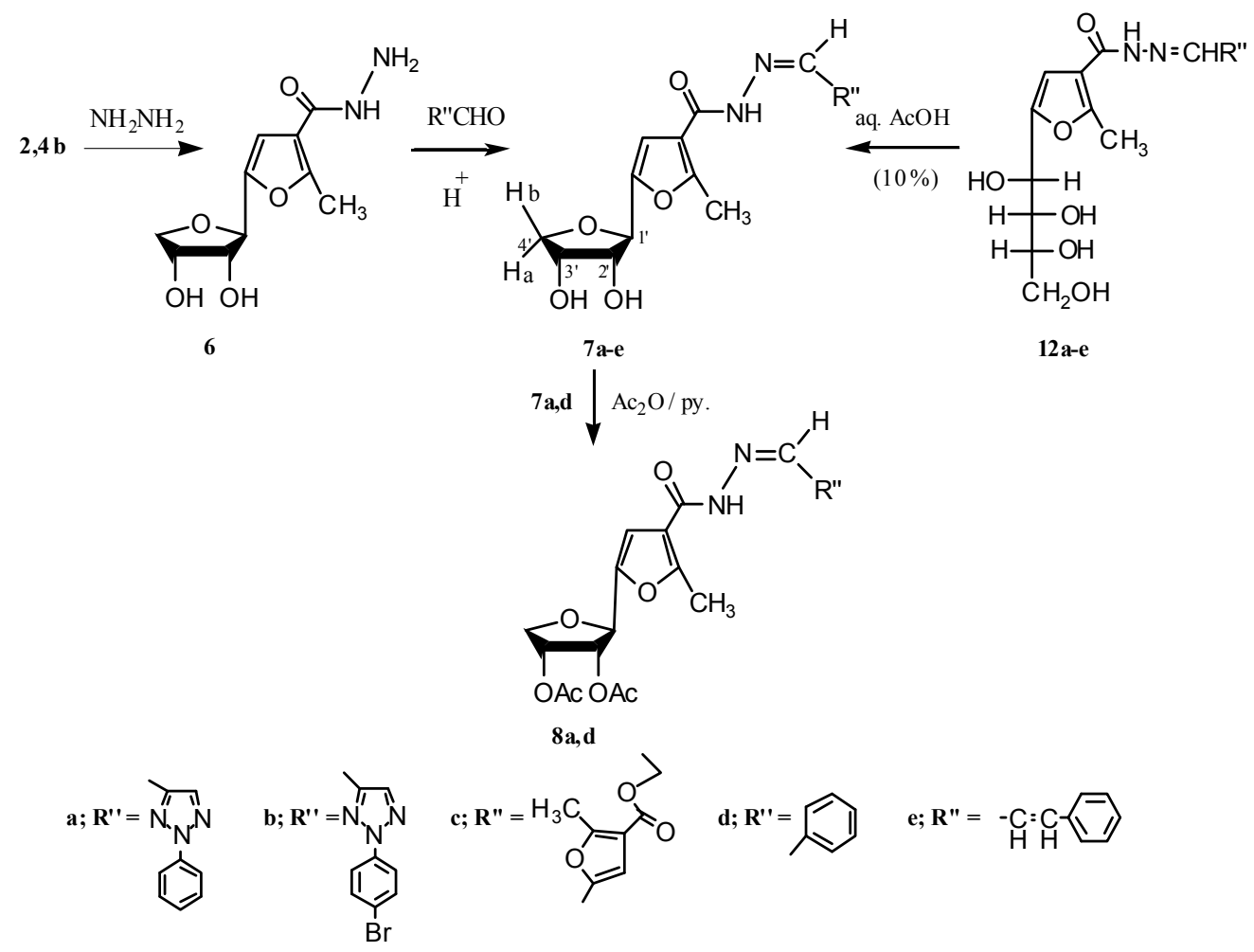

Periodate oxidation [22] of the prepared anhydro-derivatives $\mathbf{2} \mathbf{b}, \mathbf{4 b}$, gave the corresponding dialdehyde in the hemialdal structure 9 [22,23]. Furthermore, condensation of dialdehyde 9 with two molar equivalents of aroylhydrazines, afforded the corresponding bisaroylhydrazones 10a-d. ${ }^{1} \mathrm{H}-\mathrm{NMR}$ spectra of compounds 10a-d (DMSO- $\left.\mathrm{d}_{6}\right)$, showed the two $(\mathrm{NH})$ protons at $\delta(11.78-11.55)$ as two singlets, followed by the aromatic protons as a multiplet at $\delta(7.82-7.00)$, two $(\mathrm{CH}=\mathrm{N})$ as a doublet at 7.9 for $\mathrm{H}_{(2)}$ and a multiplet at 7.7 for $\mathrm{H}_{(1)}$, and the proton at position-4 in the furan ring as a singlet at $6.7 \mathrm{ppm}$. The methine proton was shown as a doublet at $\delta 5.1$, followed by a multiplet at $4.1 \mathrm{ppm}$ for the protons of the two methylene groups. Oxidative cyclization of the prepared bisaroylhydrazones $\mathbf{1 0 a}-\mathbf{d}$ and a physical and chemical study of the oxidation products, revealed that their properties could not be reconciled with that of 1,2,3-triazole derivatives $C$ [24-27] but rather was compatible with that of 1,3,4-oxadiazole derivatives 11a-d, that were obtained in appropriate yields. Infrared spectra of these compounds showed no enol benzoate group as expected for the 1,2,3-triazole derivatives $\mathrm{C}$, and showed instead a band at $1,667-1,660 \mathrm{~cm}^{-1}$, which was attributed to the conjugated carbonyl group of compounds 11a-d (see Experimental and Schemes 3 and 4).

Furthermore, ${ }^{1} \mathrm{H}-\mathrm{NMR}$ spectra $\left(\mathrm{CDCl}_{3}\right)$ of these products showed the disappearance of signals corresponding to two $(\mathrm{NH})$ and two $(\mathrm{CH}=\mathrm{N})$, methine and methylene protons. Indeed, it is noteworthy that the integration of the aromatic part $(\delta 8.22-7.36 \mathrm{ppm})$, referred to one aromatic ring only, in accord with structures $\mathbf{1 1 a}, \mathbf{b}, \mathbf{d}$. In addtion, these oxidative cyclization products displayed the proton at position-4 in the furan ring as the most downfield signal at $\delta(8.49-8.48) \mathrm{ppm}$; this proton resonated at a lower field region than that expected in $\mathrm{CDCl}_{3}$, which may be attributed to the electron withdrawing effect of the carbonyl group on the neighboring carbon atom, as well as conjugation with the phenyl oxadiazole moiety. In addition, the structure of the oxidation products $\mathbf{1 1 a}-\mathbf{d}$ was further supported 
through their boiling with hydrochloric acid, which didn't afford the corresponding amine derivatives D as expected from 1,2,3-triazole derivatives (see Experimental part and Scheme 4). Moreover, the proposed mechanism for formation of 11a-d may proceed via elimination of one aroylhydrazone part (oxidation in presence of iodine and mercuric oxide) due to the bulkiness of the molecule, followed by oxidative cyclization of the other aroylhydrazone part to afford compounds 11a-d (Scheme 5).

Scheme 3. Synthesis of bisaroylhydrazone derivatives 10a-d.

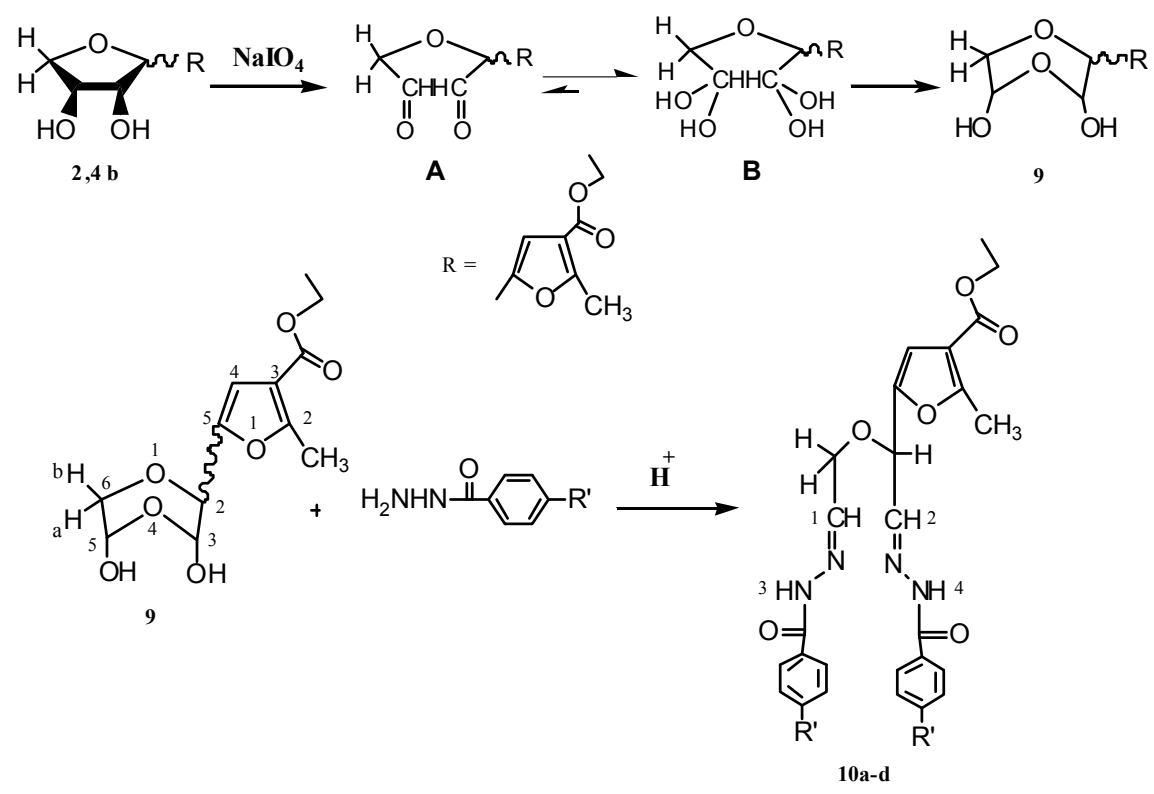

Scheme 4. Synthesis of 1,3,4-oxadiazole derivatives 11a-d.

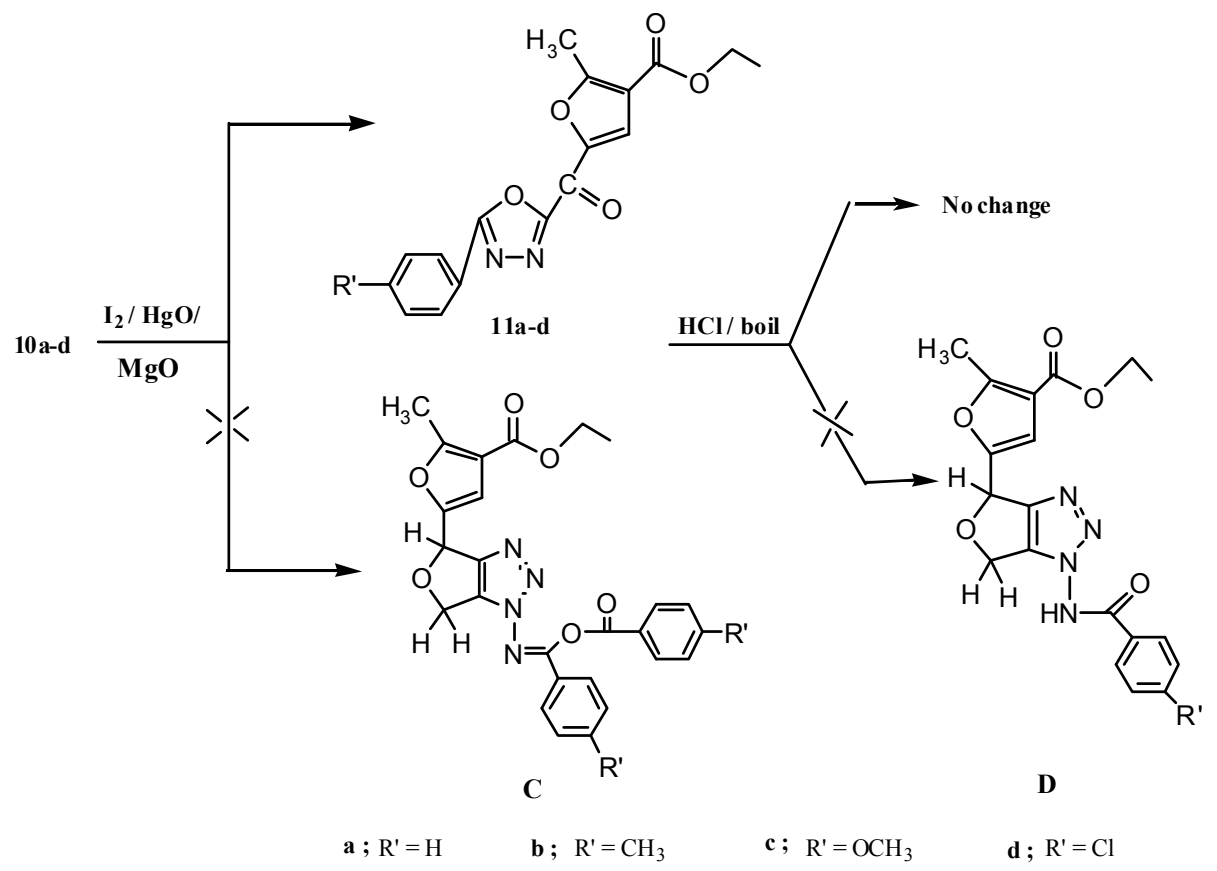


Scheme 5. Proposed mechanism for formation of ethyl 2-methyl-5-(5-aryl-1,3,4oxadiazole-2-carbonyl)furan-3-carboxylate.

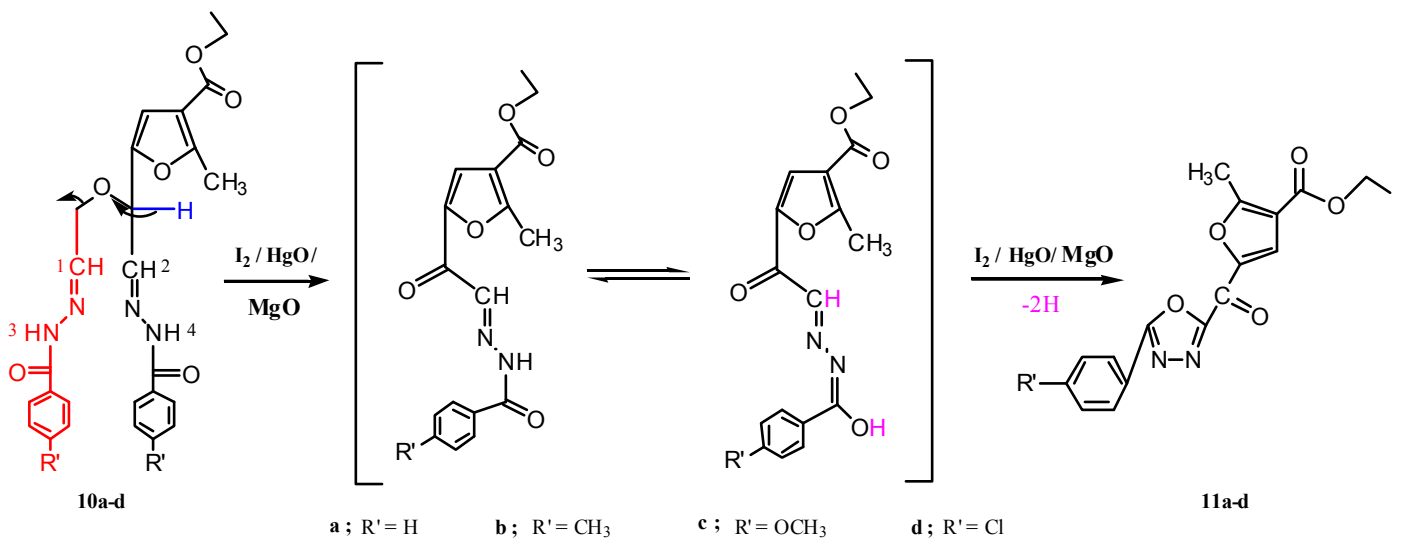

\subsection{Pharmacological Screening}

\subsubsection{MAO-B Activity}

\subsubsection{Effect of Tested Compounds on MAO-B}

This study aimed to evaluate the effect of two selected newly prepared compounds 7c,e on MAO-B activity given the biological importance of MAO-B [28-38].

\subsubsection{Determination of $\mathrm{V}_{\max }$ and $\mathrm{K}_{\mathrm{m}}$}

The $\mathrm{V}_{\max }$ and $\mathrm{K}_{\mathrm{m}}$ of the MAO-B catalyzed reaction in the presence or absence of each examined compound was carried out by plotting $\mathrm{V}$ against [S], each separately. The obtained results revealed that MAO-B was activated in the presence of compounds $\mathbf{7 c}$ and $\mathbf{7 e}$, each separately, by $6.57-$ and 6.97-fold, respectively. In addition, the MAO-B catalyzed reactions in the presence of compounds 7c and 7e have $V_{\max }$ equal to 0.49 and 0.71 , respectively. Meanwhile the $\mathrm{K}_{\mathrm{m}}$ values were 1.72 and 1.32, respectively. Our obtained data showed that compound 7e was an effective MAO-B activator, which increases the affinity of substrate to bind with the active site of MAO-B enzyme. That may be attributed to the presence of highly conjugated system with the cinamyl group in $7 \mathbf{e}$ as compared with bulky furan ring in $7 \mathbf{c}$ (Table 1, Figure 2).

Table 1. Effect of substrate concentration on the rate of MAO-B catalyzed reactions in presence of the examined compounds $7 \mathbf{c}$ and $\mathbf{7 e}$, compared to control.

\begin{tabular}{|c|c|c|c|}
\hline \multirow{2}{*}{ Substrate conc. } & \multicolumn{3}{|c|}{ Rate } \\
\cline { 2 - 4 } & Control & $\mathbf{7 c}$ & $\mathbf{7 e}$ \\
\hline $0.25 \times 10^{-3}$ & 0.020 & 0.068 & 0.222 \\
$0.50 \times 10^{-3}$ & 0.042 & 0.085 & 0.240 \\
$1.00 \times 10^{-3}$ & 0.050 & 0.151 & 0.315 \\
$1.50 \times 10^{-3}$ & 0.180 & 0.250 & 0.363 \\
$2.00 \times 10^{-3}$ & 0.200 & 0.322 & 0.440 \\
$3.00 \times 10^{-3}$ & 0.280 & 0.430 & 0.560 \\
$4.00 \times 10^{-3}$ & 0.365 & 0.481 & 0.674 \\
$5.00 \times 10^{-3}$ & 0.380 & 0.498 & 0.700 \\
\hline
\end{tabular}


Figure 2. Effect of substrate concentration on the rate of MAO-B catalyzed reactions in the presence of the examined compounds $\mathbf{7 c}, \mathbf{e}$ compared to control.

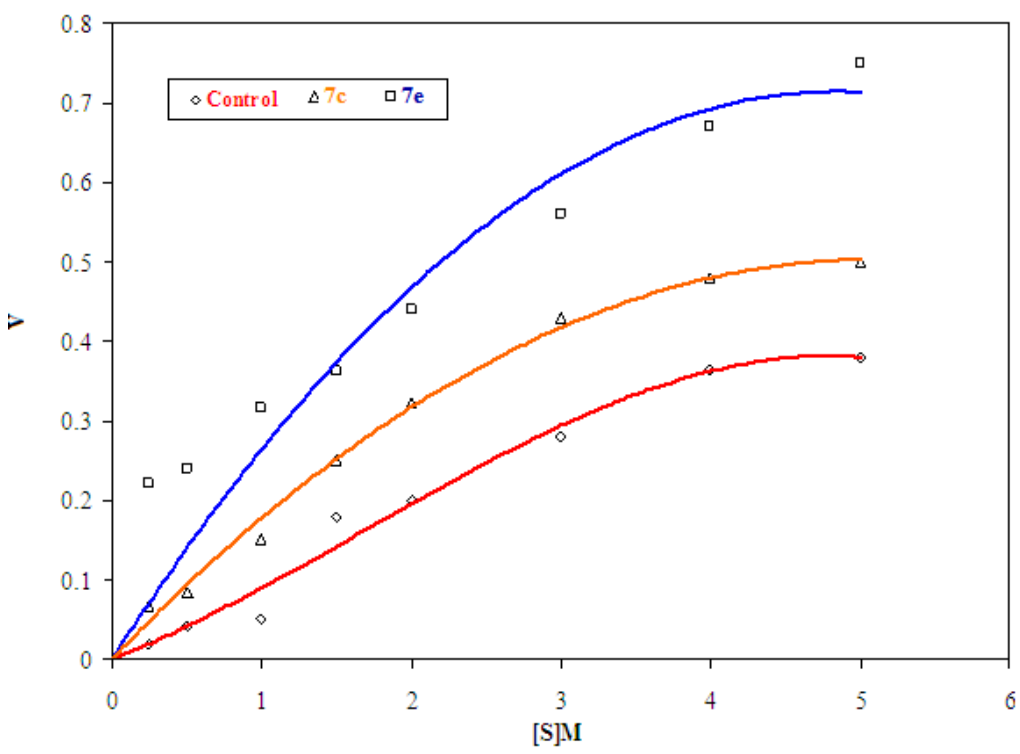

\subsubsection{Antibacterial and Antifungal Activities}

The compounds 3b, 6, 7c,e, 10a-c and 11a,b,d have been studied for their antibacterial and antifungal activities against four bacterial species (Escherichia coli, Bacillus sp., Staphylococcus sp., and Sarcina sp.) and six fungal species (Aspergillus niger, Aspergillus fmigatus, Alternaria sp., Fusarium sp., Chaetomium sp., and Penicillium sp.) using the Nutrient Agar (NA) and Sabouraud Dextrose Agar (SDA) diffusion methods, respectively, in DMSO solvent (Table 2).

Table 2. Inhibition zones of tested compounds against selected microorganisms (mm).

\begin{tabular}{|c|c|c|c|c|c|c|}
\hline \multirow{3}{*}{ Compound No. } & \multicolumn{6}{|c|}{ Bacteria } \\
\hline & \multicolumn{3}{|c|}{ Gram positive } & \multicolumn{3}{|c|}{ Gram negative } \\
\hline & Bacillus sp. & Sarcina sp. & Staphylococcus sp. & \multicolumn{3}{|c|}{ E. coli } \\
\hline $3 \mathbf{b}$ & $\ldots$ & $\ldots$ & $\ldots$ & \multicolumn{3}{|c|}{$\ldots$} \\
\hline 6 & $\ldots$ & $\ldots$ & 2 & \multicolumn{3}{|c|}{$\ldots$} \\
\hline $7 \mathrm{c}$ & $\ldots$ & $\ldots$ & 0.5 & \multicolumn{3}{|c|}{$\ldots$} \\
\hline $7 e$ & $\ldots$ & $\ldots$ & 2 & \multicolumn{3}{|c|}{$\ldots$} \\
\hline 10a & $\ldots$ & $\ldots$ & $\ldots$ & \multicolumn{3}{|c|}{$\ldots$} \\
\hline $10 \mathrm{~b}$ & $\ldots$ & $\ldots$ & $\ldots$ & \multicolumn{3}{|c|}{$\ldots$} \\
\hline $10 \mathrm{c}$ & $\ldots$ & $\ldots$ & $\ldots$ & \multicolumn{3}{|c|}{$\ldots$} \\
\hline $11 \mathrm{a}$ & $\ldots$ & $\ldots$ & 1 & \multicolumn{3}{|c|}{$\ldots$} \\
\hline $11 \mathrm{~b}$ & $\ldots$ & $\ldots$ & $\ldots$ & \multicolumn{3}{|c|}{$\ldots$} \\
\hline 11d & $\ldots$ & $\ldots$ & $\ldots$ & \multicolumn{3}{|c|}{$\ldots$} \\
\hline \multirow[b]{2}{*}{ Compound No. } & \multicolumn{6}{|c|}{ Fungi } \\
\hline & $\begin{array}{c}\text { Aspergillus } \\
\text { niger }\end{array}$ & $\begin{array}{l}\text { Aspergillus } \\
\text { fumigatus }\end{array}$ & $\begin{array}{c}\text { Alternaria } \\
\text { sp. }\end{array}$ & $\begin{array}{c}\text { Fusarium } \\
\text { sp. }\end{array}$ & $\begin{array}{c}\text { Chaetomium } \\
\text { sp. }\end{array}$ & $\begin{array}{c}\text { Penicilliu } \\
\text { sp. }\end{array}$ \\
\hline $3 \mathbf{b}$ & 1 & 1 & $\ldots$ & 2 & $\ldots$ & $\ldots$ \\
\hline 6 & $\ldots$ & $\ldots$ & 1 & 1 & 2 & 2 \\
\hline
\end{tabular}


Table 2. Cont.

\begin{tabular}{|c|c|c|c|c|c|c|}
\hline \multirow[b]{2}{*}{ Compound No. } & \multicolumn{6}{|c|}{ Fungi } \\
\hline & $\begin{array}{c}\text { Aspergillus } \\
\text { niger }\end{array}$ & $\begin{array}{l}\text { Aspergillus } \\
\text { fumigatus }\end{array}$ & $\begin{array}{c}\text { Alternaria } \\
\text { sp. }\end{array}$ & $\begin{array}{c}\text { Fusarium } \\
\text { sp. }\end{array}$ & $\begin{array}{c}\text { Chaetomium } \\
\text { sp. }\end{array}$ & $\begin{array}{c}\text { Penicilliu } \\
\text { sp. }\end{array}$ \\
\hline $7 \mathrm{c}$ & 1 & 1 & 0.5 & 2 & $\ldots$ & 0.5 \\
\hline $7 e$ & $\ldots$ & 2.5 & 0.5 & 2 & $\ldots$ & 1 \\
\hline $10 \mathrm{a}$ & $\ldots$ & $\ldots$ & $\ldots$ & $\ldots$ & $\ldots$ & $\ldots$ \\
\hline $10 \mathrm{~b}$ & 1 & $\ldots$ & $\ldots$ & $\ldots$ & $\ldots$ & $\ldots$ \\
\hline $10 \mathrm{c}$ & 1 & $\ldots$ & $\ldots$ & $\ldots$ & $\ldots$ & $\ldots$ \\
\hline $11 \mathrm{a}$ & 0.5 & $\ldots$ & 0.5 & $\ldots$ & $\ldots$ & 2 \\
\hline $11 \mathrm{~b}$ & $\ldots$ & $\ldots$ & $\ldots$ & $\ldots$ & $\ldots$ & $\ldots$ \\
\hline $11 \mathrm{~d}$ & $\ldots$ & $\ldots$ & $\ldots$ & 2 & $\ldots$ & $\ldots$ \\
\hline
\end{tabular}

....: No effect.

It was found that they were active against Gram positive bacteria (Staphylococcus sp.). In addition, the replacement of the bulky furan ring in compound 7c by the cinamyl group in compound 7e, increased the antibacterial activity against Gram positive bacteria (Staphylococcus sp.), due to the conjugation caused by the cinamyl group [39]. With respect to antifungal activity, the compounds $\mathbf{3 b}$, $\mathbf{6}, 7 \mathbf{c}, \mathbf{e}$, and $11 \mathrm{a}$ were found to be generally active against the tested fungi.

\section{Experimental}

\subsection{Chemistry}

Melting points were determined with a Melt-temp. apparatus and are uncorrected. TLC was performed on Baker-Flex silica gel 1B-F plates and the spots were detected by UV light absorption. IR spectra were recorded on Perkin Elmer. USA Spectrometer. ${ }^{1} \mathrm{H}-\mathrm{NMR},{ }^{13} \mathrm{C}-\mathrm{NMR}$, and $2 \mathrm{D}{ }^{1} \mathrm{H}-\mathrm{NMR}$ were recorded on a JEOL JNM ECA $500 \mathrm{MHz}$ instrument using tetramethylsilane as an internal standard. Mass spectra were recorded on GCMS solution DI Analysis Shimadzu Qp-2010 Plus. Optical rotation was obtained at $22{ }^{\circ} \mathrm{C}$ with a Perkin-Elmer Model 241 Polarimeter $10 \mathrm{~cm}, 1 \mathrm{~mL}$ microcell. Microanalyses were performed at the faculty of Science, Cairo University, Cairo, Egypt. Solutions were evaporated under diminished pressure unless otherwise stated. The ChemDraw-Ultra-8.0 has been used in generating the nomenclature of the prepared compounds.

\subsubsection{Ethyl 5-(2',3'-Dihydroxytetrahydrofuran-1'-yl)-2-methylfuran-3-carboxylates $\mathbf{2 b}, \mathbf{4 b}$}

Compound $1 \mathrm{~b}(15.0 \mathrm{~g}, 54.74 \mathrm{mmol})$ was boiled under reflux with aqueous acetic acid $(300 \mathrm{~mL}, 10 \%)$ for $5 \mathrm{~h}$, while the reaction was monitored by TLC (chloroform-methanol, 20:1, V/V), the starting material disappeared and a more mobile spot (Rf: 0.5 ) was obtained. The solvent was evaporated under diminished pressure and washed with toluene ( 3 times, $10 \mathrm{~mL}$ each) to obtain $\mathbf{2 b}$ as a yellow thick syrup along with a solid mass of $\mathbf{4 b}$. Separation of $\mathbf{4 b}$ from the syrup was carried out by washing the mixture with ethanol where it was partially soluble in ethanol on cold; the overall yield was $69.7 \%$; the solid mass was recrystallized from ethanol as colourless needles; IR $(\mathrm{KBr}): 3,426(\mathrm{OH}), 1,712 \mathrm{~cm}^{-1}$ (CO-ester). 


\subsubsection{Synthesis of Isopropylidene Derivatives}

General Methods. A solution of $\mathbf{2 a}, \mathbf{4 a}$ [22] or $\mathbf{2 b}, \mathbf{4 b}$ mixture $(4.42 \mathrm{mmol})$ in dry acetone (100 $\mathrm{mL})$ was treated with $p$-toluenesulphonic acid $(10.98 \mathrm{mmol})$ and stirred at room temperature. The reaction mixture was monitored by TLC (hexane-ethyl acetate, $7: 1, \mathrm{~V} / \mathrm{V}$ ), the starting material disappeared after $7 \mathrm{~h}$ and a more mobile spot appeared. The mixture was then poured onto a saturated solution of sodium bicarbonate, extracted with chloroform, the organic layer washed with water and dried over anhydrous sodium sulphate. Evaporation of the dried filtrate gave pale yellow needles.

1-[5-(2',3'-O-Isopropylidene- $\beta$-D-erythrofuranosyl)-2-methylfuran-3-yl]ethanone (3a). Yield 51\%; recrystallized from ethanol as colourless needles, mp $97-99{ }^{\circ} \mathrm{C}$; $\mathrm{R}_{\mathrm{f}}$ : 0.35 (hexane-ethyl acetate, 7:1, V/V), IR (KBr): 1,682 (CO-acetyl), 1,604, $1,571 \mathrm{~cm}^{-1}(\mathrm{C}=\mathrm{C}) ;{ }^{1} \mathrm{H}-\mathrm{NMR}\left(\mathrm{CDCl}_{3}\right) ; \delta: 1.358,1.544(2 \mathrm{~s}$,

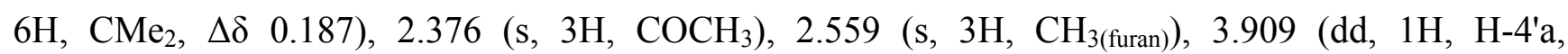
$\left.J_{3^{\prime}, 4^{\prime} \mathrm{a}}=3.85 \mathrm{~Hz}, J_{4^{\prime} \mathrm{b}, 4^{\prime} \mathrm{a}}=10.70 \mathrm{~Hz}\right), 4.049\left(\mathrm{~d}, 1 \mathrm{H}, \mathrm{H}-4{ }^{\prime} \mathrm{b}, J_{4^{\prime} \mathrm{b}, 4^{\prime} \mathrm{a}}=10.70 \mathrm{~Hz}\right), 4.892-4.937\left(\mathrm{~m}, 2 \mathrm{H}, \mathrm{H}-2^{\prime}\right.$, H-3'), 5.001 (s, 1H, H-1'), 6.475 (s, 1H, $\left.\mathrm{CH}_{\text {furan }}\right)$; MS: $m / z(\%), 267$ (3.50, $\left.\mathrm{M}^{+}+1\right), 266\left(4.82, \mathrm{M}^{+}\right), 137$ (36.26), 125 (21.36), 111 (35.32), 109 (21.18), 99 (21.56), 97 (52.51), 95 (33.70), 85 (46.86), 83 (49.75), 81 (32.98), 73 (26.34), 71 (66.24), 69 (61.30), 67 (23.40), 60 (25.40), 57 (100), 56 (21.33), 55 (65.29).

Ethyl 5-(2',3'-O-isopropylidene- $\beta$-D-erythrofuranosyl)-2-methylfuran-3-carboxylate (3b). Yield (90\%); recrystallized from ethanol as colourless needles, mp 70-72 ${ }^{\circ} \mathrm{C}$; $\mathrm{R}_{\mathrm{f}}$ : 0.63 (hexane-ethyl acetate, 7:1, $\mathrm{V} / \mathrm{V}) ;[\alpha]_{20}^{\mathrm{D}}$-69.03; IR (KBr): 1,709 (CO-ester) 1,613, 1,583 $\mathrm{cm}^{-1}(\mathrm{C}=\mathrm{C}) ;{ }^{1} \mathrm{H}-\mathrm{NMR}\left(\mathrm{CDCl}_{3}\right) ; \delta: 1.324$

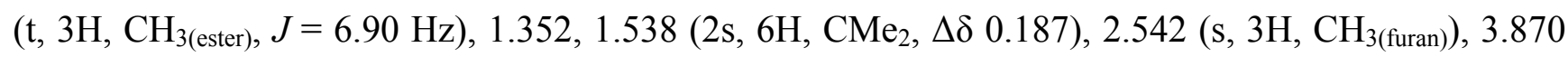
$\left(\mathrm{dd}, 1 \mathrm{H}, \mathrm{H}-4^{\prime} \mathrm{a}, J_{3^{\prime}, 4^{\prime} \mathrm{a}}=3.80 \mathrm{~Hz}, J_{4^{\prime} \mathrm{b}, 4^{\prime} \mathrm{a}}=10.70 \mathrm{~Hz}\right), 4.029\left(\mathrm{~d}, 1 \mathrm{H}, \mathrm{H}-4^{\prime} \mathrm{b}, J_{4^{\prime} \mathrm{b}, 4^{\prime} \mathrm{a}}=10.70 \mathrm{~Hz}\right), 4.260(\mathrm{q}$, $2 \mathrm{H}, \mathrm{CH}_{2 \text { (ester) }} J=6.90 \mathrm{~Hz}$ ), 4.876-4.928 (m, 2H, H-2', H-3'), 5.003 (s, 1H, H-1'), 6.486 (s, 1H, $\mathrm{CH}_{\text {furan }}$ ); MS: $m / z(\%), 297\left(6.3, \mathrm{M}^{+}+1\right), 296$ (20.6, $\left.\mathrm{M}^{+}\right), 182$ (100), 181 (30.6), 145 (60), 153 (43), 137 (25), 136 (25.6), 105 (21.3), 80 (27.5), 79 (28.8), 69 (38.1), 65 (28.8), 60 (13.8), 59 (76.3), 56 (25), 55 (30.6), 53 (33.8), 52 (44.4), $51(60), 50(25)$.

5-(2',3'-Dihydroxytetrahydrofuran-1'-yl)-2-methylfuran-3-carbohydrazide (6). A solution of $\mathbf{2 b}, \mathbf{4 b}$ (2.0 g, $7.81 \mathrm{mmol})$ in ethanol $(10 \mathrm{~mL})$ was treated with hydrazine hydrate $(4 \mathrm{~mL})$ under reflux for one hour, while the reaction was monitored by TLC (chloroform-methanol, 6:1, V/V) which revealed the absence of the starting material and formation of two more mobile spots, $\mathrm{R}_{\mathrm{f}}$ : 0.49 (major) and 0.74 (minor). After cooling 6 separated out, was filtered off, washed with a little ethanol, and dried; yield $65 \%$. It was recrystallized from ethanol as colourless needles, mp $185-187^{\circ} \mathrm{C}$. TLC showed one spot only, $\mathrm{R}_{\mathrm{f}}$ : 0.49 (chloroform-methanol, 6:1, V/V); The minor isomer was left in the mother liquor; $[\alpha]_{20}^{\mathrm{D}}$-58.98; IR (KBr): 3,384, 3,314 (OH and $\left.\mathrm{NH}_{2}\right), 3272(\mathrm{NH}), 1,636 \mathrm{~cm}^{-1}$ (CO-amide); ${ }^{1} \mathrm{H}-\mathrm{NMR}$

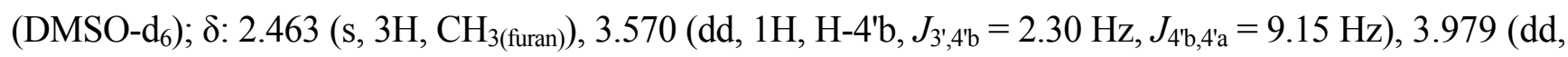
$\left.1 \mathrm{H}, \mathrm{H}-4^{\prime} \mathrm{a}, J_{3^{\prime}, 4^{\prime} \mathrm{a}}=4.60 \mathrm{~Hz}, J_{4^{\prime} \mathrm{b}, 4^{\prime} \mathrm{a}}=9.15 \mathrm{~Hz}\right), 4.029-4.066\left(\mathrm{~m}, 2 \mathrm{H}, \mathrm{H}-3^{\prime}, \mathrm{H}-2^{\prime}\right), 4.312\left(\mathrm{~s}, 2 \mathrm{H}, \mathrm{NH}_{2}\right)$, $4.395\left(\mathrm{~d}, 1 \mathrm{H}, \mathrm{H}-1^{\prime}, J_{1^{\prime}, 2^{\prime}}=6.90 \mathrm{~Hz}\right), 4.985\left(\mathrm{~d}, 1 \mathrm{H}, 2^{\prime}-\mathrm{OH}, J_{2^{\prime}, \mathrm{OH}}=4.60 \mathrm{~Hz}\right), 5.076\left(\mathrm{~d}, 1 \mathrm{H}, 3^{\prime}-\mathrm{OH}\right.$, $\left.J_{3^{\prime}, \mathrm{OH}}=6.85 \mathrm{~Hz}\right), 6.715\left(\mathrm{~s}, 1 \mathrm{H}, \mathrm{CH}_{\text {furan }}\right), 9.243(\mathrm{~s}, 1 \mathrm{H}, \mathrm{NH})$. After shaking with $\mathrm{D}_{2} \mathrm{O}$, the $\mathrm{NH}, \mathrm{NH}_{2}$, and the two hydroxyl protons disappeared; MS: $m / z(\%), 243\left(2.19, \mathrm{M}^{+}+1\right), 242\left(16.89, \mathrm{M}^{+}\right), 224(0.47$, 
$\left.\mathrm{M}^{+}-\mathrm{H}_{2} \mathrm{O}\right), 212\left(11.54, \mathrm{M}^{+}-2 \mathrm{NH}\right), 211\left(100, \mathrm{M}^{+}-\mathrm{NHNH}_{2}\right)$; Anal. Calcd for $\mathrm{C}_{10} \mathrm{H}_{14} \mathrm{~N}_{2} \mathrm{O}_{5}$ : C, 49.58; $\mathrm{H}$, 5.84 ; N, $1.55 \%$; found: C, 49.58; H, 5.83; N, $1.56 \%$.

\subsubsection{Reactions of $\mathbf{6}$ with a Number of Aldehydes}

General Methods

Method A. A solution of $6(0.5 \mathrm{~g}, 2.066 \mathrm{mmol})$ in ethanol $(5 \mathrm{~mL})$ containing acetic acid $(0.1 \mathrm{~mL})$ was treated with the corresponding aldehyde $(2.066 \mathrm{mmol})$, and the reaction mixture was refluxed on water bath for $10 \mathrm{~min}$. After cooling the product 3-carbohydrazone derivative that separated out, was filtered off, washed with little ethanol, and dried.

Method B. A solution of (1',2',3',4'-tetrahydroxybutyl)furan-3-carbohydrazone 12a-e [20,21] (4.799 mmol) was refluxed with aqueous acetic acid (10\%) for $5 \mathrm{~h}$. After cooling the compounds $7 \mathbf{a}-\mathbf{e}$ that separated out as a crystalline mass were filtered off, washed with little ethanol, and dried.

5-(2',3'-Dihydroxytetrahydrofuran-1'-yl)-2-methyl-N-[(2-phenyl-2H-1,2,3-triazol-4-yl)methyl-ene]furan3-carbohydrazone (7a). Yield of method A 87\%; recrystallized from ethanol as colourless needles, mp 209-210 ${ }^{\circ} \mathrm{C}$; $\mathrm{R}_{\mathrm{f}}$ : 0.39 (chloroform-methanol, 15:1, V/V); [ $\left.\alpha\right]_{20}^{\mathrm{D}}-37.59$; IR (KBr): 3,395 (OH), 3,108

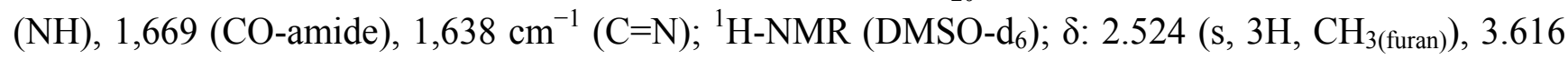
$\left(\mathrm{dd}, 1 \mathrm{H}, \mathrm{H}-44^{\prime} \mathrm{b}, J_{3^{\prime}, 4^{\prime} \mathrm{b}}=2.30 \mathrm{~Hz}, J_{4^{\prime} \mathrm{b}, 4^{\prime} \mathrm{a}}=9.20 \mathrm{~Hz}\right), 4.008-4.026$ (m, 1H, H-4'a), 4.036-4.095 (m, 2H, H-3', H-2'), 4.493 (d, 1H, H-1', $J_{1^{\prime}, 2^{\prime}}=6.90 \mathrm{~Hz}$ ), 5.043 (ss, 1H, 2'-OH), 5.142 (d, 1H, 3'-OH, $\left.J_{3^{\prime}, \mathrm{OH}}=6.15 \mathrm{~Hz}\right)$, $6.860\left(\mathrm{~s}, 1 \mathrm{H}, \mathrm{CH}_{\text {furan }}\right)$, phenyl protons: $7.432(\mathrm{t}, 1 \mathrm{H}, p-\mathrm{H}), 7.568(\mathrm{t}, 2 \mathrm{H}, O-\mathrm{H}), 8.017(\mathrm{~d}, 2 \mathrm{H}, m-\mathrm{H})$, $8.401(\mathrm{~s}, 1 \mathrm{H}, \mathrm{CH}=\mathrm{N}), 8.537\left(\mathrm{~s}, 1 \mathrm{H}, \mathrm{CH}_{\text {triazole }}\right), 11.610(\mathrm{~s}, 1 \mathrm{H}, \mathrm{NH})$. After shaking with $\mathrm{D}_{2} \mathrm{O}$, the $\mathrm{NH}$ proton and the two hydroxyl protons disappeared; MS: $m / z(\%), 399\left(0.89, \mathrm{M}^{+}+2\right), 398\left(5.36, \mathrm{M}^{+}+1\right)$, 397 (23.99, $\mathrm{M}^{+}$), 379 (3.55, $\left.\mathrm{M}^{+}-\mathrm{H}_{2} \mathrm{O}\right), 324$ (21.85), 253 (11.94, $\mathrm{M}^{+}$-phenyltriazole moiety), 211 (100), 137 (35.81), 77 (20.56, $\left.\mathrm{C}_{6} \mathrm{H}_{5}\right)$; Anal. Calcd for $\mathrm{C}_{19} \mathrm{H}_{19} \mathrm{~N}_{5} \mathrm{O}_{5}: \mathrm{C}, 57.42 ; \mathrm{H}, 4.80 ; \mathrm{N}, 17.64 \%$; found: C, 57.43; H, 4.82; N, $17.62 \%$.

$N$-[(2-(p-Bromophenyl)-2H-1,2,3-triazol-4-yl)methylene]-5-(2',3'-dihydroxytetrahydrofuran-1'-yl)-2methylfuran-3-carbohydrazone (7b). Yield of method A 84\%; recrystallized from ethanol as colourless needles, mp 241-243 ${ }^{\circ} \mathrm{C}$; $\mathrm{R}_{\mathrm{f}}$ : 0.38 (chloroform-methanol, 15:1, V/V); [ $\left.\alpha\right]_{20}^{\mathrm{D}}-31.39$; IR (KBr): 3,423 (OH), 3,249 (NH), 1,653 (CO-amide), 1,618 $\mathrm{cm}^{-1}(\mathrm{C}=\mathrm{N}) ;{ }^{1} \mathrm{H}-\mathrm{NMR}$ (DMSO-d $)$; $\delta: 2.519$ (s, 3H, $\mathrm{CH}_{3(\text { furan) }}$ ), 3.617 (dd, 1H, H-4'b, $J_{3^{\prime}, 4^{\prime} \mathrm{b}}=2.30 \mathrm{~Hz}, J_{4^{\prime} \mathrm{b}, 4^{\prime} \mathrm{a}}=9.95 \mathrm{~Hz}$ ), 4.019 (dd, 1H, H-4'a, $\left.J_{3^{\prime}, 4^{\prime} \mathrm{a}}=3.85 \mathrm{~Hz}, J_{4^{\prime} \mathrm{b}, 4^{\prime} \mathrm{a}}=9.95 \mathrm{~Hz}\right), 4.080-4.109$ (m, 2H, H-3', H-2'), 4.493 (d, 1H, H-1', $\left.J_{1^{\prime}, 2^{\prime}}=6.90 \mathrm{~Hz}\right)$, $5.051\left(\mathrm{ss}, 1 \mathrm{H}, 2^{\prime}-\mathrm{OH}\right), 5.147\left(\mathrm{~d}, 1 \mathrm{H}, 3^{\prime}-\mathrm{OH}, J_{3^{\prime}, \mathrm{OH}}=6.10 \mathrm{~Hz}\right), 6.857\left(\mathrm{~s}, 1 \mathrm{H}, \mathrm{CH}_{\text {furan }}\right), p$-bromophenyl protons: $7.752(\mathrm{~d}, 2 \mathrm{H}, m-\mathrm{H}), 7.954(\mathrm{~d}, 2 \mathrm{H}, o-\mathrm{H}), 8.422(\mathrm{~s}, 1 \mathrm{H}, \mathrm{CH}=\mathrm{N}), 8.518\left(\mathrm{~s}, 1 \mathrm{H}, \mathrm{CH}_{\text {(triazole })}\right)$, $11.630(\mathrm{~s}, 1 \mathrm{H}, \mathrm{NH})$. After shaking with $\mathrm{D}_{2} \mathrm{O}$, the $\mathrm{NH}$ proton and the two hydroxyl protons disappeared; ${ }^{13} \mathrm{C}-\mathrm{NMR} ; \delta$ : 14.00 (C-13), 70.91 (C-4'), 73.32 (C-3'), 75.33 (C-2'), 76.50 (C-1'), 107.79 (C-12), 115.11 (C-11), 120.92 (C-10), 121.23 (C-9), 133.27 (C-8), 135.16 (C-7), 137.74 (C-6), 138.52 (C-5), 146.50 (C-4), 151.71 (C-3), 158.19 (C-2), 159.84 (C-1); MS: $m / z$ (\%), 478/476 (2.03, 2.17, $\left.\mathrm{M}^{+}+1\right), 477 / 475\left(8.85,9.10, \mathrm{M}^{+}\right), 459 / 457\left(1.25,1.24, \mathrm{M}^{+}-\mathrm{H}_{2} \mathrm{O}\right), 253$ (10.01, $\mathrm{M}^{+}-p$-bromophenyl triazole moiety), 211 (100), 137 (34); Anal. Calcd for $\mathrm{C}_{19} \mathrm{H}_{18} \mathrm{BrN}_{5} \mathrm{O}_{5}$ : C, 47.90; H, 3.83; N, 14.71; $\mathrm{Br}, 16.77 \%$; found: $\mathrm{C}, 47.91 ; \mathrm{H}, 3.81 ; \mathrm{N}, 14.70 ; \mathrm{Br}, 16.78 \%$. 
Ethyl 5-[(2-(5-(2',3'-dihydroxytetrahydrofuran-1'-yl)-2-methylfuran-3-carbonyl)hydrazono)-methyl]-2methylfuran-3-carboxylate (7c). Yield of method A 93\%; [ $\alpha]_{20}^{\mathrm{D}}-44.42$; IR (KBr): 3,426 (OH), 3,208 (NH), 1,694 (CO-ester), 1,655 (CO-amide), 1,616 cm ${ }^{-1}(\mathrm{C}=\mathrm{N}) ;{ }^{1} \mathrm{H}-\mathrm{NMR}$ (DMSO-d $\left.\mathrm{d}_{6}\right) ; \delta$ : 1.254 (t, 3H,

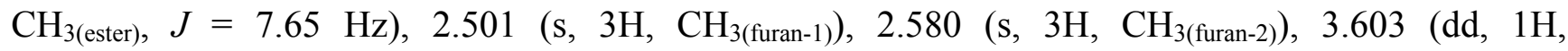
H-4'b, $\left.J_{3^{\prime}, 4^{\prime} \mathrm{b}}=2.30 \mathrm{~Hz}, J_{4^{\prime} \mathrm{b}, 4^{\prime} \mathrm{a}}=9.90 \mathrm{~Hz}\right), 4.004\left(\mathrm{dd}, 1 \mathrm{H}, \mathrm{H}-4^{\prime} \mathrm{a}, J_{3^{\prime}, 4^{\prime} \mathrm{a}}=4.60 \mathrm{~Hz}, J_{4^{\prime} \mathrm{b}, 4^{\prime} \mathrm{a}}=9.90 \mathrm{~Hz}\right)$,

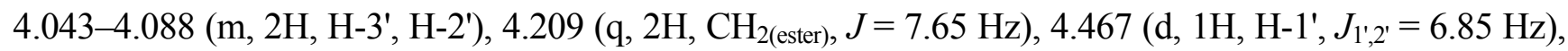
$5.028\left(\mathrm{~d}, 1 \mathrm{H}, 2^{\prime}-\mathrm{OH}, J_{2^{\prime}, \mathrm{OH}}=3.80 \mathrm{~Hz}\right), 5.124\left(\mathrm{~d}, 1 \mathrm{H}, 3^{\prime}-\mathrm{OH}, J_{3^{\prime}, \mathrm{OH}}=6.10 \mathrm{~Hz}\right), 6.830\left(\mathrm{~s}, 1 \mathrm{H}, \mathrm{CH}_{\text {furan- } 1}\right)$, $7.059\left(\mathrm{~s}, 1 \mathrm{H}, \mathrm{CH}_{\text {furan-2}}\right), 8.150(\mathrm{~s}, 1 \mathrm{H}, \mathrm{CH}=\mathrm{N}), 11.409(\mathrm{~s}, 1 \mathrm{H}, \mathrm{NH})$. After shaking with $\mathrm{D}_{2} \mathrm{O}$, the $\mathrm{NH}$ proton and the two hydroxyl protons disappeared; MS: $m / z(\%), 408\left(0.79, \mathrm{M}^{+}+2\right), 407\left(4.16, \mathrm{M}^{+}+1\right)$, 406 (18.26, $\mathrm{M}^{+}$), 211 (100), 154 (32.75); Anal. Calcd for $\mathrm{C}_{19} \mathrm{H}_{22} \mathrm{~N}_{2} \mathrm{O}_{8}$ : C, 56.13; H, 5.47; N, 6.86\%; found: $\mathrm{C}, 56.15 ; \mathrm{H}, 5.46 ; \mathrm{N}, 6.89 \%$.

N-Benzylidene-5-(2',3'-dihydroxytetrahydrofuran-1'-yl)-2-methylfuran-3-carbohydrazone (7d). Yield of method A 84\%; recrystallized from ethanol as colourless needles, mp $86-88{ }^{\circ} \mathrm{C}$; $\mathrm{R}_{\mathrm{f}}$ : 0.345 (chloroform-methanol, 15:1, V/V); [ $\alpha]_{20}^{\mathrm{D}}-42.16$; IR (KBr): 3,380 (OH and NH), 1,661 (CO-amide), $1,616 \mathrm{~cm}^{-1}(\mathrm{C}=\mathrm{N}) ;{ }^{1} \mathrm{H}-\mathrm{NMR}\left(\mathrm{DMSO}-\mathrm{d}_{6}\right) ; \delta: 2.513$ (s, 3H, $\mathrm{CH}_{3(\text { furan })}$ ), 3.608 (dd, $1 \mathrm{H}, \mathrm{H}-4^{\prime} \mathrm{b}, J_{3^{\prime}, 4^{\prime} \mathrm{b}}=2.30 \mathrm{~Hz}$, $\left.J_{4^{\prime} \mathrm{b}, 4^{\prime} \mathrm{a}}=9.95 \mathrm{~Hz}\right), 4.012\left(\mathrm{dd}, 1 \mathrm{H}, \mathrm{H}-4^{\prime} \mathrm{a}, J_{3^{\prime}, 4^{\prime} \mathrm{a}}=4.60 \mathrm{~Hz}, J_{4^{\prime} \mathrm{b}, 4^{\prime} \mathrm{a}}=9.95 \mathrm{~Hz}\right), 4.065-4.089$ (m, 2H, H-3', H-2'), $4.476\left(\mathrm{~d}, 1 \mathrm{H}, \mathrm{H}-1^{\prime}, J_{1^{\prime}, 2^{\prime}}=6.90 \mathrm{~Hz}\right), 5.043\left(\mathrm{~d}, 1 \mathrm{H}, 2^{\prime}-\mathrm{OH}, J_{2^{\prime}, \mathrm{OH}}=3.85 \mathrm{~Hz}\right), 5.141\left(\mathrm{~d}, 1 \mathrm{H}, 3^{\prime}-\mathrm{OH}\right.$, $\left.J_{3^{\prime}, \mathrm{OH}}=6.10 \mathrm{~Hz}\right), 6.869\left(\mathrm{~s}, 1 \mathrm{H}, \mathrm{CH}_{(\mathrm{furan})}\right)$, phenyl protons: $7.395-7.436(\mathrm{~m}, 3 \mathrm{H}, p-\mathrm{H}, O-\mathrm{H}), 7.672(\mathrm{~d}$, $2 \mathrm{H}, m-\mathrm{H}), 8.340(\mathrm{~s}, 1 \mathrm{H}, \mathrm{CH}=\mathrm{N}), 11.389(\mathrm{~s}, 1 \mathrm{H}, \mathrm{NH})$. After addition of $\mathrm{D}_{2} \mathrm{O}$, the $\mathrm{NH}$ proton and the two hydroxyl protons disappeared; MS: $m / z(\%), 331\left(2.25, \mathrm{M}^{+}+1\right), 330\left(10.80, \mathrm{M}^{+}\right), 227(9.18$, $\mathrm{M}^{+}-\mathrm{C}_{6} \mathrm{H}_{5} \mathrm{CN}$ ), 211 (100), 154 (59.63), 77 (9.32, $\mathrm{C}_{6} \mathrm{H}_{5}$ ); Anal. Calcd for $\mathrm{C}_{17} \mathrm{H}_{18} \mathrm{~N}_{2} \mathrm{O}_{5}$ : C, 61.80; H, 5.50; $\mathrm{N}, 8.45 \%$; found: C, $61.8 ; \mathrm{H}, 5.49 ; \mathrm{N}, 8.48 \%$.

5-(2',3'-Dihydroxytetrahydrofuran-1'-yl)-2-methyl-N-(3-phenylallylidene)furan-3-carbohydrazone (7e). Yield of method A 92.5\%; recrystallized from ethanol as colourless needles, mp $162-163{ }^{\circ} \mathrm{C}$; $\mathrm{R}_{\mathrm{f}}$ : 0.78 (chloroform:-methanol, 10:1, V/V); [ $\alpha]_{20}^{\mathrm{D}}-42.55$; IR (KBr): 3,409 (OH), 3,241 (NH), 1,657 (CO-amide), $1,628 \mathrm{~cm}^{-1}(\mathrm{C}=\mathrm{N}) ;{ }^{1} \mathrm{H}-\mathrm{NMR}\left(\mathrm{DMSO}_{6}\right) ; \delta: 2.499$ (s, 3H, $\left.\mathrm{CH}_{3 \text { (furan) }}\right), 3.598-3.617$ (m, 1H, H-4'b), $4.013\left(\mathrm{dd}, 1 \mathrm{H}, \mathrm{H}-4^{\prime} \mathrm{a}, J_{3^{\prime}, 4^{\prime} \mathrm{a}}=3.80 \mathrm{~Hz}, J_{4^{\prime} \mathrm{b}, 4^{\prime} \mathrm{a}}=9.90 \mathrm{~Hz}\right), 4.026-4.104$ (m, 2H, H-3', H-2'), 4.476 (d, 1H, $\left.\mathrm{H}-1^{\prime}, J_{1^{\prime}, 2^{\prime}}=6.90 \mathrm{~Hz}\right), 5.025\left(\mathrm{~d}, 1 \mathrm{H}, 2^{\prime}-\mathrm{OH}, J_{2^{\prime}, \mathrm{OH}}=3.80 \mathrm{~Hz}\right), 5.121\left(\mathrm{~d}, 1 \mathrm{H}, 3^{\prime}-\mathrm{OH}, J_{3^{\prime}, \mathrm{OH}}=6.15 \mathrm{~Hz}\right)$; $6.849\left(\mathrm{~s}, 1 \mathrm{H}, \mathrm{CH}_{\text {furan }}\right), 7.00(\mathrm{bs}, 2 \mathrm{H}, \mathrm{CH}=\mathrm{CH})$, phenyl protons: $7.273-7.302(\mathrm{~m}, 1 \mathrm{H}, p-\mathrm{H}), 7.355(\mathrm{t}, 2 \mathrm{H}$, $O-\mathrm{H}), 7.585(\mathrm{~d}, 2 \mathrm{H}, m-\mathrm{H}) ; 8.124(\mathrm{~d}, 1 \mathrm{H}, \mathrm{CH}=\mathrm{N}, J=6.15 \mathrm{~Hz}), 11.264(\mathrm{~s}, 1 \mathrm{H}, \mathrm{NH}) ; \mathrm{MS}: m / z(\%), 358$ $\left(0.21, \mathrm{M}^{+}+2\right), 357\left(0.95, \mathrm{M}^{+}+1\right), 356\left(3.22, \mathrm{M}^{+}\right), 338\left(0.11, \mathrm{M}^{+}-\mathrm{H}_{2} \mathrm{O}\right), 320\left(0.21, \mathrm{M}^{+}-2 \mathrm{H}_{2} \mathrm{O}\right), 279$ (0.52, $\left.\mathrm{M}^{+}-\mathrm{C}_{6} \mathrm{H}_{5}\right), 211(100), 154$ (55.50), 130 (35.56), 77 (8.65, $\left.\mathrm{C}_{6} \mathrm{H}_{5}\right), 43$ (49, $\left.\mathrm{COCH}_{3}\right)$; Anal. Calcd for $\mathrm{C}_{19} \mathrm{H}_{20} \mathrm{~N}_{2} \mathrm{O}_{5}$ : C, 64.03; H, 5.64; N, 7.84\%; found: C, 64.04; H, 5.66; N, 7.86\%.

\subsubsection{Reactions of Compounds 7a,d with Acetic Anhydride}

General Method: A solution of the sugar derivative $7 \mathbf{a}$ or $\mathbf{7 d}(1.51 \mathrm{mmol})$ in a mixture of pyridine $(15 \mathrm{~mL})$ and acetic anhydride $(15 \mathrm{~mL})$ was kept overnight at room temperature with occasional shaking. Then it was poured onto crushed ice, the di- $O$-acetyl derivative that separated out, was filtered off, washed with water, and dried. 
l'-[2-Methyl-3-(2-((2-phenyl-2H-1,2,3-triazol-4-yl)methylene)hydrazinecarbonyl)furan-5-yl]-tetra-hydro furan-2',3'-diyl diacetate (8a). Yield 76.5\%; recrystallized from dilute ethanol as colourless needles, mp 179-181 ${ }^{\circ} \mathrm{C} ; \mathrm{R}_{\mathrm{f}} 0.5$ (hexane-ethyl acetate, 2:1, V/V); [ $\left.\alpha\right]_{20}^{\mathrm{D}}-47.75 ; \mathrm{IR}(\mathrm{KBr}): 3,217(\mathrm{NH}), 1,754$ (OAc), 1,647 (CO-amide), 1,601 cm ${ }^{-1}(\mathrm{C}=\mathrm{N}) ;{ }^{1} \mathrm{H}-\mathrm{NMR}\left(\mathrm{CDCl}_{3}\right) ; \delta: 2.061,2.139$ (2s, 6H, 2OAc),

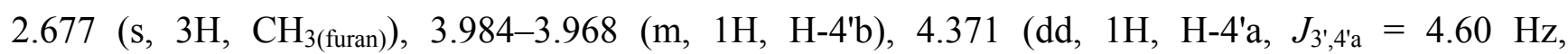
$\left.J_{4^{\prime} \mathrm{b}, 4^{\prime} \mathrm{a}}=9.95 \mathrm{~Hz}\right), 4.925\left(\mathrm{~d}, 1 \mathrm{H}, \mathrm{H}-1^{\prime}, J_{1^{\prime}, 2^{\prime}}=6.10 \mathrm{~Hz}\right), 5.492-5.532\left(\mathrm{~m}, 2 \mathrm{H}, \mathrm{H}-3^{\prime}, \mathrm{H}-2^{\prime}\right), 6.744(\mathrm{bs}, 1 \mathrm{H}$, $\left.\mathrm{CH}_{\text {furan }}\right), 7.462-7.478(\mathrm{~m}, 1 \mathrm{H}, p-\mathrm{H}), 7.531-7.625(\mathrm{~m}, 2 \mathrm{H}, O-\mathrm{H}), 8.019-8.141(\mathrm{~m}, 4 \mathrm{H}, m-\mathrm{H}, \mathrm{CH}=\mathrm{N}$, $\left.\mathrm{CH}_{\text {triazole }}\right)$, and 12.239 (s, $\left.1 \mathrm{H}, \mathrm{NH}\right)$; MS: $m / z(\%), 482\left(0.61, \mathrm{M}^{+}+1\right), 481\left(1.97, \mathrm{M}^{+}\right), 363(22.58), 362$ (100), 295 (67.47), 137 (21.53), 115 (19.79), 77 (15.78); Anal. Calcd for $\mathrm{C}_{23} \mathrm{H}_{23} \mathrm{~N}_{5} \mathrm{O}_{7}$ : C, 57.35; H, 4.82 ; N, 14.53\%; found: C, 57.38; H, 4.82; N, $14.55 \%$.

1'-[3-(2-Benzylidenehydrazinecarbonyl)-2-methylfuran-5-yl] tetrahydrofuran-2',3'-diyl diacetate (8d). Yield 80\%; recrystallized from dilute methanol as colourless needles, $\mathrm{mp} 81-82{ }^{\circ} \mathrm{C} ; \mathrm{R}_{\mathrm{f}} 0.5$ (hexane-ethyl acetate, 2:1, V/V); $[\alpha]_{20}^{\mathrm{D}}-48.37$; IR (KBr): 3,248 (NH), 1,752 (OAc), 1,654 (CO-amide), 1,582 $\mathrm{cm}^{-1}$

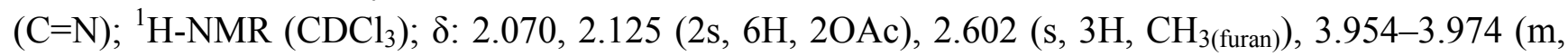
1H, H-4'b), 4.381-4.350 (m, 1H, H-4'a), 4.950 (bs, 1H, H-1'), 5.417-5.524 (m, 2H, H-3', H-2'), 6.620 (s, $\left.1 \mathrm{H}, \mathrm{CH}_{\text {furan }}\right), 7.519-7.549(\mathrm{~m}, 5 \mathrm{H}$, phenyl protons), $7.883(\mathrm{~d}, 1 \mathrm{H}, \mathrm{CH}=\mathrm{N}, J=7.65 \mathrm{~Hz}), 9.550$ (s, 1H, NH); MS: $m / z(\%), 415\left(0.39, \mathrm{M}^{+}+1\right), 414\left(0.81, \mathrm{M}^{+}\right), 295$ (100), 77 (2.61); Anal. Calcd for $\mathrm{C}_{21} \mathrm{H}_{22} \mathrm{~N}_{2} \mathrm{O}_{7}$ : C, 60.86; H, 5.37; N, 6.77\%; found: C, 60.86; H, 5.35; N, 6.76\%.

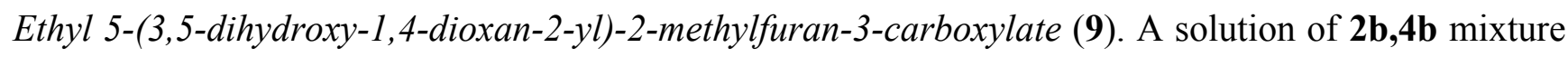
$(12.96 \mathrm{~g}, 50.62 \mathrm{mmol})$ in distilled water $(20 \mathrm{~mL})$ was treated dropwise with a solution of sodium metaperiodate $(10.825 \mathrm{~g}, 50.62 \mathrm{mmol})$ in distilled water $(20 \mathrm{~mL})$ with continuous stirring for $3 \mathrm{~h}$. The dialdehyde that separated out was filtered off, washed with little water, and dried, yield $67 \% ; \mathrm{R}_{\mathrm{f}}: 0.38$ (chloroform-methanol, 15:1, V/V). Recrystallized from ethanol as colourless needles, mp $111-113{ }^{\circ} \mathrm{C}$ (Lit. [22], $\left.111-113{ }^{\circ} \mathrm{C}\right)$; IR ( $\left.\mathrm{KBr}\right): 3,426(\mathrm{OH}), 1,712 \mathrm{~cm}^{-1}$ (CO-ester); ${ }^{1} \mathrm{H}-\mathrm{NMR}\left(\mathrm{CDCl}_{3}\right) ; \delta$ : $1.293-1.326$

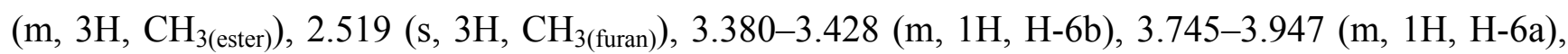

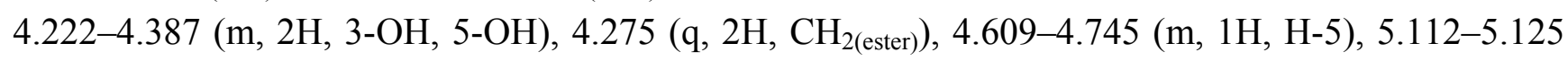
(m, 1H, H-3), 5.514-5.534 (m, 1H, H-2), and 6.677 (s, 1H, $\left.\mathrm{CH}_{\text {furan }}\right)$.

\subsubsection{Reactions of 9 with a Number of Aroylhydrazines}

General Method: A solution of $9(2.0 \mathrm{~g}, 7.353 \mathrm{mmol})$ in ethanol $(10 \mathrm{~mL})$ containing acetic acid $(0.1 \mathrm{~mL})$ was treated with the corresponding aroylhydrazine $(14.71 \mathrm{mmol})$ in ethanol $(10 \mathrm{~mL})$. The reaction mixture was refluxed on water bath for $15 \mathrm{~min}$, the bisaroylhydrazone derivative that separated out was filtered off, washed with little ethanol, and dried.

Ethyl 5-[2-(2-benzoylhydrazono)-1-(2-(2-benzoylhydrazono)ethoxy)ethyl]-2-methylfuran-3-carboxylate (10a). Yield 61\%; recrystallized from ethanol-chloroform as colourless needles, mp $204-206{ }^{\circ} \mathrm{C}$; $\mathrm{R}_{\mathrm{f}}$ : 0.55 (chloroform-methanol, 10:1, V/V); IR (KBr): 3,280, 3,197 (2NH), 1,715 (CO-ester), 1,658 (CO-amide), $1,591 \mathrm{~cm}^{-1}(\mathrm{C}=\mathrm{N}) ;{ }^{1} \mathrm{H}-\mathrm{NMR}\left(\mathrm{DMSO}_{\mathrm{d}}\right)$; $\delta: 1.223\left(\mathrm{t}, 3 \mathrm{H}, \mathrm{CH}_{3(\text { ester })}, J=6.90 \mathrm{~Hz}\right), 2.524$

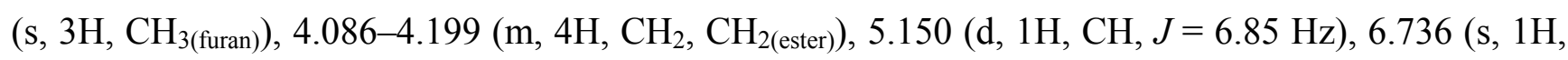
$\mathrm{CH}_{\text {furan }}$ ), phenyl protons: 7.472-7.476 (m, 4H, $\left.O-\mathrm{H}\right), 7.550-7.546(\mathrm{~m}, 2 \mathrm{H}, p-\mathrm{H}), 7.794-7.837(\mathrm{~m}, 4 \mathrm{H}$, 
$m-\mathrm{H}), 7.719$ (bs, $\left.1 \mathrm{H}, \mathrm{CH}=\mathrm{N}_{1}\right), 7.912\left(\mathrm{~d}, 1 \mathrm{H}, \mathrm{CH}=\mathrm{N}_{2}, J=6.10 \mathrm{~Hz}\right), 11.673\left(\mathrm{~s}, 1 \mathrm{H}, \mathrm{NH}_{3}\right), 11.777(\mathrm{~s}, 1 \mathrm{H}$, $\left.\mathrm{NH}_{4}\right)$; MS: $m / z(\%), 472\left(0.57, \mathrm{M}^{+}-\mathrm{H}_{2} \mathrm{O}\right), 354$ (56.88), 181 (31.16), 173 (29.44), 153 (23.60), 147 (93.31), 121 (41.45), 106 (49.34), 105 (100), 104 (23.78), 78 (21.08), 77 (99.13), 51 (77.47), 50 (24.02); Anal. Calcd for $\mathrm{C}_{26} \mathrm{H}_{26} \mathrm{~N}_{4} \mathrm{O}_{6}$ : C, 63.65; H, 5.35; N, 11.43\%; found: C, 63.66; H, 5.34; N, 11.42\%.

Ethyl 5-[2-(2-(p-toluoyl)hydrazono)-1-(2-(2-(p-toluoyl)hydrazono)ethoxy)ethyl]-2-methylfuran-3-carboxylate (10b). Yield 58\%; recrystallized from ethanol-chloroform as colourless needles, mp $204-206{ }^{\circ} \mathrm{C}$; $\mathrm{R}_{\mathrm{f}}$ : 0.22 (chloroform-methanol, 15:1, V/V); IR (KBr): 3,305, 3,183 (2NH), 1,717 (CO-ester), 1,651 (CO-amide), 1,625, 1,612 $\mathrm{cm}^{-1}(\mathrm{C}=\mathrm{N}) ;{ }^{1} \mathrm{H}-\mathrm{NMR}$ (DMSO-d $)$ ); $\delta: 1.222$ (t, 3H, $\mathrm{CH}_{3 \text { (ester) }}, J=6.85 \mathrm{~Hz}$ ),

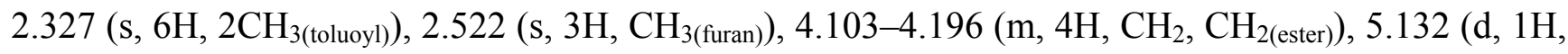
$\mathrm{CH}, J=6.90 \mathrm{~Hz}), 6.727\left(\mathrm{~s}, 1 \mathrm{H}, \mathrm{CH}_{\text {furan }}\right)$, aromatic protons: $7.265-7.280(\mathrm{~m}, 4 \mathrm{H}, O-\mathrm{H}), 7.708-7.747(\mathrm{~m}$, $\left.5 \mathrm{H}, m-\mathrm{H}, \mathrm{CH}=\mathrm{N}_{1}\right), 7.899\left(\mathrm{~d}, 1 \mathrm{H}, \mathrm{CH}=\mathrm{N}_{2}, J=6.10 \mathrm{~Hz}\right), 11.601\left(\mathrm{~s}, 1 \mathrm{H}, \mathrm{NH}_{3}\right), 11.716\left(\mathrm{~s}, 1 \mathrm{H}, \mathrm{NH}_{4}\right)$; MS: $m / z(\%), 518\left(0.09, \mathrm{M}^{+}\right), 500\left(0.18, \mathrm{M}^{+}-\mathrm{H}_{2} \mathrm{O}\right), 368$ (31.86), 161 (44.53), 161 (44.53), 119 (100), 91 (50.53), 65 (19.49); Anal. Calcd for $\mathrm{C}_{28} \mathrm{H}_{30} \mathrm{~N}_{4} \mathrm{O}_{6}$ : C, 64.87; H, 5.85; N, 10.78\%; found: C, 64.85; $\mathrm{H}, 5.83 ; \mathrm{N}, 10.80 \%$.

Ethyl 5-[2-(2-(p-anisoyl)hydrazono)-1-(2-(2-(p-anisoyl)hydrazono)ethoxy)ethyl]-2-methylfuran-3carboxylate (10c). Yield 57\%; recrystallized from ethanol-chloroform as colourless needles, $\mathrm{mp}$ 204-206 ${ }^{\circ} \mathrm{C}$; $\mathrm{R}_{\mathrm{f}}$ : 0.32 (chloroform:-methanol, 10:1, V/V); IR (KBr): 3,268, 3,212 (2NH), 1,718 (CO-ester), 1,645 (CO-amide), $1,610 \mathrm{~cm}^{-1}(\mathrm{C}=\mathrm{N}) ;{ }^{1} \mathrm{H}-\mathrm{NMR}$ (DMSO-d $\left.\mathrm{d}_{6}\right) ; \delta: 1.225$ (t, 3H, $\mathrm{CH}_{3 \text { (ester) }}, J=6.15 \mathrm{~Hz}$ ),

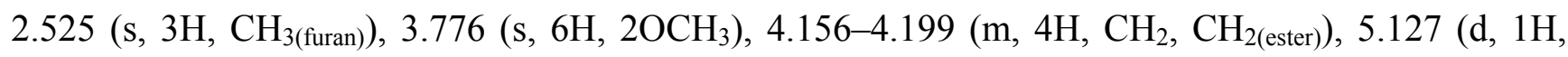
$\mathrm{CH}, J=6.15 \mathrm{~Hz}), 6.726\left(\mathrm{~s}, 1 \mathrm{H}, \mathrm{CH}_{\text {furan }}\right)$, aromatic protons: 6.700-7.008 (m, 4H, $\left.m-\mathrm{H}\right), 7.799-7.837$ $(\mathrm{m}, 4 \mathrm{H}, O-\mathrm{H}), 7.707\left(\mathrm{bs}, 1 \mathrm{H}, \mathrm{CH}=\mathrm{N}_{1}\right), 7.895\left(\mathrm{~d}, 1 \mathrm{H}, \mathrm{CH}=\mathrm{N}_{2}, J=6.10 \mathrm{~Hz}\right), 11.554\left(\mathrm{~s}, 1 \mathrm{H}, \mathrm{NH}_{3}\right)$, $11.653\left(\mathrm{~s}, 1 \mathrm{H}, \mathrm{NH}_{4}\right)$; MS: $m / z(\%), 532\left(0.15, \mathrm{M}^{+}-\mathrm{H}_{2} \mathrm{O}\right), 135$ (100); Anal. Calcd for $\mathrm{C}_{28} \mathrm{H}_{30} \mathrm{~N}_{4} \mathrm{O}_{8}$ : C, 61.05; H, 5.47; N, 10.19\%; found: C, 61.08; H, 5.49; N, 10.18\%.

Ethyl 5-[2-(2-(p-chlorobenzoyl)hydrazono)-1-(2-(2-(p-chlorobenzoyl)hydrazono)ethoxy)ethyl]-2methylfuran-3-carboxylate (10d). Yield 62\%; recrystallized from ethanol-chloroform as colourless needles, mp 204-206 ${ }^{\circ}$; Rf: 0.4 (chloroform-methanol, 15:1, V/V); IR (KBr): 3,270, 3,215 (2NH), 1,719 (CO-ester), 1,650 (CO-amide), $1,612 \mathrm{~cm}^{-1}(\mathrm{C}=\mathrm{N})$; Anal. Calcd for $\mathrm{C}_{26} \mathrm{H}_{24} \mathrm{Cl}_{2} \mathrm{~N}_{4} \mathrm{O}_{6}$ : C, 55.80; H, 4.33; N, 10.02; Cl, 12.69\%; found: C, 55.82; H, 4.32; N, 10.02; Cl, 12.68\%.

\subsubsection{Reactions of Compounds 10a-d with Yellow Mercuric Oxide}

General Method: A solution of 10a-d $(4.5 \mathrm{mmol})$ in dry ether $(50 \mathrm{~mL})$ was stirred with yellow mercuric oxide $(3.0 \mathrm{~g})$, magnesium oxide $(0.3 \mathrm{~g})$, and iodine $(2.5 \mathrm{~g})$ at room temperature for $48 \mathrm{~h}$ under anhydrous condition. The reaction mixture was filtered off, and the filtrate washed with potassium iodide solution, sodium thiosulphate, and water, respectively, then dried over anhydrous sodium sulphate. On evaporation of the dried filtrate a yellow syrup was obtained, which was crystallized from ethanol. An additional crop was obtained by extracting the inorganic residue with chloroform which upon concentration and dilution with ethanol yielded the same product. 
Ethyl 2-methyl-5-(5-phenyl-1,3,4-oxadiazole-2-carbonyl)furan-3-carboxylate (11a). Yield 36\%; recrystallized from ethanol as colourless needles, $\mathrm{mp} 168-170{ }^{\circ} \mathrm{C}$; $\mathrm{R}_{\mathrm{f}}$ : 0.41 (hexane-ethyl acetate, 6:1, V/V); IR (KBr): $1,715\left(\right.$ CO-ester), 1,660 $(\mathrm{C}=\mathrm{O}), 1,594 \mathrm{~cm}^{-1}(\mathrm{C}=\mathrm{N}) ;{ }^{1} \mathrm{H}-\mathrm{NMR}\left(\mathrm{CDCl}_{3}\right) ; \delta: 1.391$ (t,

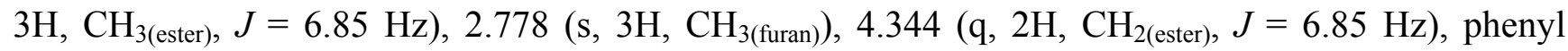
protons: $7.565(\mathrm{t}, 2 \mathrm{H}, O-\mathrm{H}), 7.611-7.637(\mathrm{~m}, 1 \mathrm{H}, p-\mathrm{H}), 8.210-8.224(\mathrm{~m}, 2 \mathrm{H}, m-\mathrm{H}), 8.491(\mathrm{~s}, 1 \mathrm{H}$, $\left.\mathrm{CH}_{\text {furan }}\right)$; MS: $m / z(\%), 328\left(3.07, \mathrm{M}^{+}+2\right), 327\left(20.48, \mathrm{M}^{+}+1\right), 326\left(100, \mathrm{M}^{+}\right), 181$ (22.15), 153 (32.73), 145 (61.44), 77 (49.04); Anal. Calcd for $\mathrm{C}_{17} \mathrm{H}_{14} \mathrm{~N}_{2} \mathrm{O}_{5}$ : C, 62.57; H, 4.32; N, 8.59\%; found: C, 62.57; $\mathrm{H}, 4.33 ; \mathrm{N}, 8.60 \%$.

Ethyl 2-methyl-5-(5-(p-tolyl)-1,3,4-oxadiazole-2-carbonyl)furan-3-carboxylate (11b). Yield 39\%; recrystallized from ethanol as colourless needles, $\mathrm{mp} 185-187^{\circ} \mathrm{C}$; $\mathrm{R}_{\mathrm{f}}$ : 0.52 (hexane-ethyl acetate, 6:1, $\mathrm{V} / \mathrm{V})$; IR (KBr): 1,715 (CO-ester), 1,660 (C=O), 1,611 cm ${ }^{-1}(\mathrm{C}=\mathrm{N}) ;{ }^{1} \mathrm{H}-\mathrm{NMR}\left(\mathrm{CDCl}_{3}\right) ; \delta: 1.390(\mathrm{t}$,

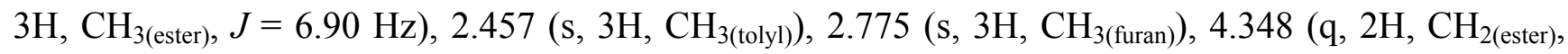
$J=6.90 \mathrm{~Hz}$ ), phenyl protons: $7.361(\mathrm{~d}, 2 \mathrm{H}, O-\mathrm{H}), 8.098$ (d, 2H, $m-\mathrm{H}), 8.482\left(\mathrm{~s}, 1 \mathrm{H}, \mathrm{CH}_{\text {furan }}\right)$; Anal. Calcd for $\mathrm{C}_{18} \mathrm{H}_{16} \mathrm{~N}_{2} \mathrm{O}_{5}$ : C, 63.53; H, 4.76; N, 8.25\%; found: C, 63.52; H, 4.74; N, 8.23\%.

Ethyl 2-methyl-5-[5-(p-anisyl)-1,3,4-oxadiazole-2-carbonyl]furan-3-carboxylate (11c). Yield 21\%; recrystallized from ethanol as colourless needles, $\mathrm{mp} 182-183{ }^{\circ} \mathrm{C}$; $\mathrm{R}_{\mathrm{f}}$ : 0.41 (hexane-ethyl acetate, 6:1, V/V); IR (KBr): 1,714 (CO-ester), 1,657 (C=O), 1,606 cm $\mathrm{cm}^{-1}(\mathrm{C}=\mathrm{N})$; Anal. Calcd for $\mathrm{C}_{18} \mathrm{H}_{16} \mathrm{~N}_{2} \mathrm{O}_{6}$ : C, 60.66; H, 4.55; N, 7.86\%; found: C, 60.67; H, 4.53; N, 7.86\%.

Ethyl 2-methyl-5-[5-(p-chlorophenyl)-1,3,4-oxadiazole-2-carbonyl]furan-3-carboxylate (11d). Yield $39 \%$; recrystallized from ethanol as colourless needles, $\mathrm{mp} 177-178{ }^{\circ} \mathrm{C}$; $\mathrm{R}_{\mathrm{f}}$ : 0.59 (hexane-ethyl acetate, 6:1, V/V); IR (KBr): 1,723 (CO-ester), 1,667 (C=O), and 1,600 $\mathrm{cm}^{-1}(\mathrm{C}=\mathrm{N})$; ${ }^{1} \mathrm{H}-\mathrm{NMR}$

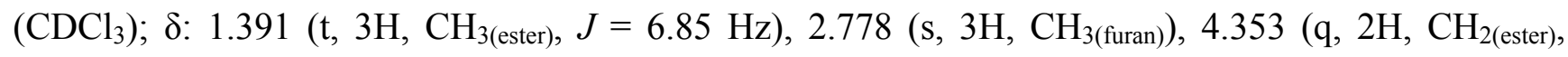
$J=6.85 \mathrm{~Hz}), p$-chlorophenyl protons: $7.550(\mathrm{~d}, 2 \mathrm{H}, m-\mathrm{H}), 8.158(\mathrm{~d}, 2 \mathrm{H}, O-\mathrm{H}), 8.482\left(\mathrm{~s}, 1 \mathrm{H}, \mathrm{CH}_{\text {furan }}\right)$; ${ }^{1} \mathrm{H}-\mathrm{NMR}\left(\mathrm{DMSO}_{6}\right.$ ); $\delta: 1.293$ (t, 3H, $\left.\mathrm{CH}_{3(\text { ester) }}, J=6.85 \mathrm{~Hz}\right), 2.692$ (s, 3H, $\mathrm{CH}_{3(\text { furan })}$ ), 4.269 (q, 2H, $\left.\mathrm{CH}_{2 \text { (ester) }}, J=6.85 \mathrm{~Hz}\right), p$-chlorophenyl protons: $7.700(\mathrm{~d}, 2 \mathrm{H}, m-\mathrm{H}), 8.082(\mathrm{~d}, 2 \mathrm{H}, O-\mathrm{H}), 8.170(\mathrm{~s}, 1 \mathrm{H}$, $\left.\mathrm{CH}_{\text {furan }}\right)$; MS: $m / z(\%), 362 / 360$ (100, 42, $\left.\mathrm{M}^{+}\right), 181$ (78.7), 179 (71.29), 153 (78.02), 141/139 (11.12, 33.56), 137 (39.6), $113 / 111$ (10.89, 33.86); Anal. Calcd for $\mathrm{C}_{17} \mathrm{H}_{13} \mathrm{ClN}_{2} \mathrm{O}_{5}$ : C, 56.62; H, 3.64; N, 7.75; $\mathrm{Cl}, 9.81 \%$; found: $\mathrm{C}, 56.60 ; \mathrm{H}, 3.63 ; \mathrm{N}, 7.77 ; \mathrm{Cl}, 9.83 \%$.

\subsection{Pharmacological Screening}

\subsubsection{MAO-B Activity}

\subsubsection{Enzyme Preparation}

Rabbit brain was homogenized in 9 volumes of ice-cold $0.1 \mathrm{M}$ sodium phosphate buffer ( $\mathrm{pH} 7.4$ ) with an Ultra-Turrax 18/2 homogenizer. The separated mitochondrial fraction was suspended in phosphate buffer to give a final volume of $1 \mathrm{~mL} \mathrm{~g}^{-1}$ weight of tissue [40]. 


\subsubsection{Enzyme Assay}

Monoamine oxidase-B activity was assayed in presence and absence of the examined compounds each separately using benzylamine as substrate by continuous recording on a Pye Unicam SP8-100 Double Beam Spectrophotometer of the increase in extinction at $250 \mathrm{~nm}$ produced at $38{ }^{\circ} \mathrm{C}$.

\subsubsection{Determination of $\mathrm{V}_{\max }$ and $\mathrm{K}_{\mathrm{m}}$}

The $\mathrm{V}_{\max }$ and $\mathrm{K}_{\mathrm{m}}$ of MAO-B catalyzed reaction in presence or absence of each examined compound 7c and 7e was carried out by plotting the rate of the reaction (V) against substrate concentration [S], each separately.

\subsubsection{Antibacterial and Antifungal Screening}

The antibacterial and antifungal activities of synthesized compounds $3 \mathbf{b}, \mathbf{6}, \mathbf{7 c}, \mathbf{e}, \mathbf{1 0 a}-\mathbf{c}$ and $\mathbf{1 1 a}, \mathbf{b}, \mathbf{d}$ against four bacterial species (Escherichia coli, Bacillus sp., Staphylococcus sp., and Sarcina sp.) and six fungal species (Aspergillus niger, Aspergillus fmigatus, Alternaria sp., Fusarium sp., Chaetomium sp., and Penicillium sp.). have been studied by using the Nutrient Agar (NA) and Sabouraud Dextrose Agar (SDA) diffusion methods, respectively in DMSO solvent. The bacteria were subcultured on Nutrient Agar medium (NA), whereas, fungi were subcultured on Sabouraud Dextrose Agar (SDA). The composition is given in $\mathrm{g} / \mathrm{L}$ unless otherwise stated. The $\mathrm{pH}$ value of the media was adjusted to $7 \pm 0.1$ prior to sterilization with $0.1 \mathrm{M}$ sodium hydroxide or hydrochloric acid. All media were prepared with distilled water and sterilized by autoclaving at $121^{\circ} \mathrm{C}$ for $20 \mathrm{~min}$. Nutrient Agar (NB): Peptone, 5; beef extract, 3; NaCl, 5; agar, 20. Sabouraud Dextrose Agar (SDA): Peptone, 10; glucose, 40; agar, 20. The stock solutions $(1 \mathrm{mg} / \mathrm{mL})$ of the test chemicals were prepared by dissolving $10 \mathrm{mg}$ of the test compounds in $10 \mathrm{~mL}$ dimethylsulphoxide (DMSO) solvent. Petri plates $(150 \mathrm{~mm} \times 15 \mathrm{~mm})$ were prepared by pouring $60 \mathrm{~mL}$ of SDA and allowed to solidify. Plates were dried and $1 \mathrm{~mL}$ of each standardized inoculums suspension was poured and uniformly spread. The excess inoculums was drained and the inoculums was allowed to dry for $15 \mathrm{~min}$. Eight equidistant wells were made in the medium using a sterile cork borer $(6 \mathrm{~mm}$ in diameter and $50 \mu \mathrm{L}$ of the test chemicals $(1 \mathrm{mg} / \mathrm{mL})$ diluted in DMSO 2\% were placed into the wells. The Petri-dishes containing bacterial and fungi species were incubated at $37^{\circ} \mathrm{C}$ for $24 \mathrm{~h}$, and $48 \mathrm{~h}$, respectively. The tests were carried in triplicate. The antimicrobial activity was measured as the diameter $(\mathrm{mm})$ of clear zone of growth inhibition.

\section{Conclusions}

Some new $C$-glycoside derivatives and heterocyclic derivatives of carbohydrate have been prepared and their physical and biological properties were studied. It was found that compounds with highly conjugated systems are acting as MAO-B activators, as well as antibacterial agents, meanwhile all tested compounds have antifungal activities. 


\section{Acknowledgments}

Thanks are due to Sallam, M.A., Faculty of Science, Alexandria University for optical rotation measurements, and El-Badn, D., Department of Botany, Faculty of Science, Alexandria University for microbiological studies.

\section{References and Notes}

1. Rainier, J.D.; Cox, J.M. Aluminum- and boron-mediated $C$-glycoside synthesis from 1,2-anhydroglycosides. Org. Lett. 2000, 2, 2707-2709.

2. Yale, H.L.; Losee, K. 2-Amino-5-substituted 1,3,4-oxadiazoles and 5-imino-2-substituted $\Delta^{2}$-1,3,4-oxadiazolines. A group of novel muscle relaxants. J. Med. Chem. 1966, 9, 478-483.

3. Omar, F.A.; Mahfouz, N.M.; Rahman, M.A. Design, synthesis and anti-inflammatory activity of some 1,3,4-oxadiazole derivatives. Eur. J. Med. Chem. 1996, 31, 819-825.

4. Palusa, S.K.G.; Udupi, R.H.; Himabindu, V.; Sridhara, A.M. Synthesis and evaluation of a series of pyrimidine substituted 1,3,4-oxadiazole derivatives as antimicrobial and anti-inflammatory agents. Org. Commun. 2011, 4, 82-93.

5. Tan, T.M.C.; Chen, Y.; Kong, K.H.; Bai, J.; Li, Y.; Lim, S.G.; Ang, T.H.; Lam, Y. Synthesis and the biological evaluation of 2-benzenesulfonyl alkyl-5-substituted-sulfanyl-(1,3,4)-oxadiazoles as potential anti-hepatitis B virus agents, Antiviral Res. 2006, 71, 7-14.

6. Adelstein, G.W.; Yen, C.H.; Dajani, E.Z.; Bianchi, R.G. 3,3-Diphenyl-3-(2-alkyl-1,3,4-oxadiazol5-yl)propylcycloalkylamines, a novel series of antidiarrheal agents, J. Med. Chem. 1976, 19, $1221-1225$.

7. Jha, K.K.; Samad, A.; Kumar, Y.; Shaharyar, M.; Khosa, R.; Jain, J.; Bansal, S. 3D-QSAR studies of 1,3,4-oxadiazole derivatives as antimycobacterial agents. Iran. J. Pharm. Res. 2009, 8, 163-167.

8. Macaev, F.; Rusu, G.; Pogrebnoi, S.; Gudima, A.; Stingaci, E.; Vlad, L.; Shvets, N.; Kandemirli, F.; Dimoglo, A.; Reynolds, R. Synthesis of novel 5-aryl-2-thio-1,3,4-oxadiazoles and the study of their structure-anti-mycobacterial activities. Bioorg. Med. Chem. 2005, 13, 4842-4850.

9. Navarrete-Vazquez, G.; Molina-Salinas, G.M.; Duarte-Fajardo, Z.V.; Vargas-Villarreal, J.; Estrada-Soto, S.; Gonzalez-Salazar, F.; Hernandez-Nunez, E.; Said-Fernandez, S. Synthesis and antimycobacterial activity of 4-(5-substituted-1,3,4-oxadiazol-2-yl)pyridines, Bioorg. Med. Chem. 2007, 15, 5502-5508.

10. Zarghi, A.; Tabatabai, S.A.; Faizi, M.; Ahadian, A.; Navabi, P.; Zanganeh, V.; Shafiee, A. Synthesis and anticonvulsant activity of new 2-substituted-5-(2-benzyloxyphenyl)-1,3,4-oxadiazoles. Bioorg. Med. Chem. Lett. 2005, 15, 1863-1865.

11. Jin, L.; Chen, J.; Song, B.; Chen, Z.; Yang, S.; Li, Q.; Hu, D.; Xu, R. Synthesis, structure, and bioactivity of $N^{\prime}$-substituted benzylidene-3,4,5-trimethoxybenzohydrazide and 3-acetyl-2 substituted phenyl-5-(3,4,5-trimethoxyphenyl)-2,3-dihydro-1,3,4-oxadiazole derivatives. Bioorg. Med. Chem. Lett. 2006, 16, 5036-5040.

12. Sawant, R.; Lanke, P.; Jadhav, G.; Bhangale, L. QSAR analysis of structurally similar 1,3,4-oxadiazoles as enzyme tyrosinase inhibitors. Drug Invent. Today 2010, 2, 169-172. 
13. Sánchez, A.G.; Roldán, A.R. Dehydraion of 2-(D-arabino-tetrahyroxybutyl)furans. Carbohydr. Res. 1972, 22, 53-62.

14. González, F.G. Reactions of monosaccharides with beta-ketonic esters and related substances. In Advances in Carbohydrate Chemistry; Wolfrom, L.M., Tipson, S.R., Eds.; Academic Press Inc.: New York, NY, USA, 1956; Volume 11, pp. 97-143.

15. El-Sallam, M.A.; Hegazy, E.I.A. Studies on 3-epimeric 2-hexulose phenylosazones. Structure and anomeric configuration of the 3,6-anhydro-osazone derivatives obtained from D-arabino- and D-ribo-2-hexulose phenylosazone. Carbohydr. Res. 1981, 95, 177-188.

16. El-Sallam, M.A.; Ibrahim, E.S.I.; El-Eter, K.A.A.; Cassady, J.M. Synthesis and anomeric configuration of 2-(erythrofuranosyl)benzimidazole $C$-nucleoside analogues. Carbohydr. Res. 1997, 298, 93-104.

17. Sable, H.Z.; Ritchey, W.M.; Nordlander, J.E. Studies on cyclic polyols part IV. Nuclear magnetic resonance spectra and configurational assignments of polyhydroxycyclopentanes. Carbohydr. Res. 1965, $1,10-21$.

18. MacCoss, M.; Robins, M.J.; Rayner, B.; Imbach, J.L. A new aspect of the use of 2',3'-O-isopropylidene ribonucleosides for investigation of anomeric configuration. Carbohydr. Res. 1977, 59, 575-579.

19. Maeba, I.; Iwata, K.; Usami, F.; Furukawa, H. C-Nucleosides 1. Synthesis of 3-( $\beta$ - $D-$-ribofuranosyl) pyridazines. J. Org. Chem. 1983, 48, 2998-3002.

20. Hassan, S.Y.; Yaccout, G.A.; Abd El-Dayem, N.S.; El Sadek, M.M. Chemistry Department, Faculty of Science, Alexandria University, Alexandria, Egypt. Unpublished work, 2009.

21. El Sadek, M.M.; Zagzoug, N.B.; El Soccary, N.N. Reactions of 2-methyl-5-(D-arabinotetrahydroxybutyl)-3-furoylhydrazine. Carbohydr. Res. 1993, 250, 323-326.

22. Sánchez, A.G.; Roldán, A.R. Dehydration of 2-(D-arabino-tetrahydroxybutyl)furans. Carbohydr. Res. 1972, 22, 53-62.

23. Guthrie, R.D.; Honeyman, J. Periodate oxidation of methyl 4:6- $O$-benzylidene- $\alpha$-D-glucoside. Chem. Ind. 1958, 29, 388-389.

24. Alexandrou, N.E. Reassignment of structures of the dihydro-v-tetrazines. II: Mechanism of oxidation of diacylhydrazones. Tetrahedron 1966, 22, 1309-1316.

25. El Khadem, H.; Shaban, M.A.E. Carbohydrate derivatives of 1-substituted 1,2,3-triazoles. J. Chem. Soc. C 1967, 1967, 519-520.

26. El-Khadem, H.; Shaban, M.A.E.; Nassr, M.A.M. Oxidation of mesoxalaldehyde bis- and tris-(benzoylhydrazones). J. Chem. Soc. C 1969, 1969, 1416-1418.

27. El-Khadem, H.; El-Sadik, M.M.; Meshreki, M.H. Reactions of phenylglyoxal bisarylhydrazones. J. Chem. Soc. C 1968, 1968, 2097-2099.

28. Ni, X.; Chan, D.; Chan, K.; McMain, S.; Kennedy, J.L. Serotonin genes and gene-gene interactions in borderline personality disorder in a matched case-control study. Prog. Neuropsychopharmacol. Biol. Psychiatry 2009, 33, 128-133.

29. Upadhyay, A.K.; Edmondson, D.E. Characterization of detergent purified recombinant rat liver monoamine oxidase B expressed in Pichia pastoris. Protein Expr. Purif. 2008, 59, 349-356.

30. Setini, A.; Pierucci, F.; Senatori, O.; Nicotra, A. Molecular characterization of monoamine oxidase in zebrafish (Danio rerio). Comp. Biochem. Physiol. 2005, 140, 153-161. 
31. Kaneda, Y.; Fujii, A.; Nagamine, I. Platelet serotonin concentrations in medicated schizophrenic patients. Prog. Neuropsychopharmacol. Biol. Psychiatry 2001, 25, 983-992.

32. Garpenstrand, H.; Longato-Stadler, E.; Klinteberg, B.A.; Grigorenko, E.; Damberg, M.; Oreland, L.; Hallman, J. Low platelet monoamine oxidase activity in Swedish imprisoned criminal offenders. Eur. Neuropsychopharmacol. 2002, 12, 135-140.

33. Parsian, A.; Racette, B.; Zhang, Z.H.; Rundle, M.; Perlmutter, J.S. Association of variations in monoamine oxidases A and B with Parkinson's disease subgroups. Genomics 2004, 83, 454-460.

34. Paris, J.; Zweig-Frank, H.; Ying Kin, N.M.K.N.; Schwartz, G.; Steiger, H.; Nair, N.P.V. Neurobiological correlates of diagnosis and underlying traits in patients with borderline personality disorder compared with normal controls. Psychiatry Res. 2004, 121, 239-252.

35. Vindis, C.; Séguélas, M.H.; Bianchi, P.; Parini, A.; Cambon, C. Monoamine oxidase B induces ERK-dependent cell mitogenesis by hydrogen peroxide generation. Biochem. Biophys. Res. Commun. 2000, 271, 181-185.

36. Carpéné, C.; Daviaud, D.; Boucher, J.; Bour, S.; Visentin, V.; Grès, S.; Duffaut, C.; Fontana, E.; Testar, X.; Saulnier-Blache, J.S.; et al. Short- and long-term insulin-like effects of monoamine oxidases and semicarbazide-sensitive amine oxidase substrates in cultured adipocytes. Metabolism 2006, 55, 1397-1405.

37. Rommelspacher, H.; Meier-Henco, M.; Smolka, M.; Kloft, C. The levels of norharman are high enough after smoking to affect monoamineoxidase B in platelets. Eur. J. Pharmachol. 2002, 441, 115-125.

38. Petzer, J.P.; Castagnoli, N., Jr; Schwarzschild, M.A.; Chen, J.F.; Schyf, C.J.V.D. Dual-target-directed drugs that block monoamine oxidase $\mathrm{B}$ and adenosine $\mathrm{A}_{2 \mathrm{~A}}$ receptors for Parkinson's disease. Neurotherapeutics 2009, 6, 141-151.

39. Rahman, M.A. Chalcone: A valuable insight into the recent advances and potential pharmacological activities. Chem. Sci. J. 2011, 2011, CSJ-29.

40. Green, A.L.; El Hait, M.A.S. p-Methoxyamphetamine, a potent reversible inhibitor of type-A monoamine oxidase in vitro and in vivo. J. Pharm. Pharmacol. 1980, 32, 262-266.

Sample Availability: Samples of the compounds are available from the authors.

(C) 2012 by the authors; licensee MDPI, Basel, Switzerland. This article is an open access article distributed under the terms and conditions of the Creative Commons Attribution license (http://creativecommons.org/licenses/by/3.0/). 